\title{
Capillary Wave Scattering From a Surfactant Domain
}

\author{
T. Chou \\ Department of Physics, Harvard University, Cambridge, MA 02138 \\ S. K. Lucas and H. A. Stone \\ Division of Applied Sciences, Harvard University, Cambridge, MA 02138
}

(May 6, 2019)

\begin{abstract}
The study of capillary wave scattering by a circular region with different interfacial properties from the rest of an otherwise homogeneous interface is motivated by experiments on wave attenuation at a monolayer covered air-water interface where domains of one surface phase are dispersed in a second surface phase. Here the scattering function is calculated for an incident wave of frequency $\omega$ (wavevector $k_{0}$ ) scattering from an isolated circular domain of radius $a$ with surface tension $\sigma_{1}$ which is imbedded in an otherwise infinite interface of surface tension $\sigma_{0}$. The underlying fluid is treated as irrotational and the three-dimensional flow problem coupling the heterogeneous surface to the underlying liquid is reduced to a set of dual integral equations, which are solved numerically. With this solution the scattering amplitudes and the total scattering cross sections are calculated as a function of the surface tension ratio $\sigma_{0} / \sigma_{1}$ and incident wavenumber $k_{0} a$. The analogous problem of a discontinuous change in bending rigidity is also considered and the solution to the complete viscous problem is outlined in an appendix. Experimental implications of these results are discussed.
\end{abstract}

\section{INTRODUCTION}

The interfacial region separating two distinct fluids has significant dynamical effects on many transport processes. In particular, fluctuations in the interface shape are present in problems as diverse as classical propagation of capillarygravity waves, fluctuations of cell membranes, and mass transport across the ocean surface. The majority of analyses of such surface waves have treated a fluctuating interface with homogeneous properties. Nevertheless, due to the typical phase-separatedstructure of monolayer covered interfaces or the presence of impurities, the surface may in fact be heterogeneous.1.2 For example, interfacial tension or bending rigidity, two material properties used to characterize the interfacial resistance to deformation, may vary along an interface. In this paper, we analyze the effect of a circular domain of different interfacial properties on the propagation of a capillary wave and so deduce the scattering characteristics.

A typical feature of surface fluctuations with wavelength $\lambda$ is that the velocity in the surrounding fluid may be disturbed to a distance $\lambda$ with a magnitude proportional to the magnitude of the restoring force. Hence, in the neighborhood of a substantial change in surface properties, it is likely that local transport processes are affected. A first approach to understanding the dynamics of a heterpgeneous interface is to describe the mechanism of a plane wave scattering from an isolated surface inhomogeneity. 3 However, almost all previous experimental and theoretical research on surface wave scattering has focysed on the effects gf solid bodies in contact with the bulk fluid 1 e.g. scattering producedby nonuniform bottoms 6 , floating objects 5 , and solid structures such as cylinders protruding from the interface 0 These studies have concentrated on the gravity wave limit with a few also treating capillary wave propagation. There is a rich mathematical literature describing gravity wave scattering by floating surface objects (such as a circular disk) with papers dating back 40 years.

There are now many experimental observations, including surfactant systems and lipid monolayers, of the complex, heterogeneous surface structures that develop when amphiphilic molecules reside at an air-water interface 1 , 2 Frequently, the interface consists of circular domains (with radii tens of microns ranging up to a centimeter) of one phase interspersed in a second phase with different surface concentration. The surfactant concentration differences locally change the parameters which govern the stress balance at the interface. Both the surface tension and bending rigidity of the interface can thus be altered and in these small mesoscopic systems, it is surface tension, or bending rigidity, rather than gravity, that provides the dominant restoring force to surface deformations. Therefore, we study capillary wave propagation in the presence of an isolated circular island with different surface properties from the surrounding interface. Our work provides an example of wave scattering due to a boundary condition heterogeneity.

Experiments on capillary wave propagation at fluid-gas inferfaces covered with surfactants can show a dramatic increase in the damping rate of the surface capillary waves.10 15 In these experiments, a wave maker generates a capillary wave and either the dispersion relation, wave amplitude, or the width of the spectral peaks generated from light scattering of a thermally fluctuating interface, is measured, and the damping coefficient characterizing the attenuation of the interface deformation is extracted. The damping coefficient is observed to depend on the surface 
coverage and appears to have a maximum near the regime where surface phases coexist. 1015 The damping rate may also depend on the typical domain sizes which coexist in the monolayer.11 Since the damping rates may be five times greater than that due fo viscous damping, a mechanism including the effects of wave scattering by surface domains has been suggested, 16 but there is as yet no proven explanation for the substantial damping rate increase.

Boundary conditions for gravity waves do not involve higher mixed derivatives and as gravity acts uniformly along the interface, surface wave scattering may be produced by imposed boundary conditions, e.g. a fixed dock occupying a half-plane on an infinite free surface. Such problems have been studied with Wiener-Hopf techniques 17 Furthermore, wave scattering from an intrinsic boundary condition heterogeneity can occur when a varying interfacial tension or rigidity is present. In this case, the higher mixed derivatives that occur in the boundary conditions couple to the nonuniform surface parameters to produce surface wave scattering. This type of problem has received little attention. In particular, approaches to this latter problem in the past have been approximate, one dimensional geometry with additional assumptions concerning the stability of the problem.18

Here, we study analytically surface wave scattering from a circular discontinuity in surface tension or bending rigidity. In Section II, we outline the boundary value problem for an irrotational subphase. Hankel transforms may be used to describe the velocity potential and application of the normal stress balance at the heterogeneous interface leads to a system of dual integral equations with Bessel function kernels. This system is solved numerically. The analogous problem accounting for viscous effects is described in an appendix. For a benchmark comparison, we also derive results for scattering from a stretched, free membrane with an imbedded region of different tension. In Section III, we consider capillary wave scattering from a circular region of different interfacial bending rigidity and compare the results to bending wave scattering along a membrane with a circular inclusion of different flexural rigidity. The results of these different models are discussed and compared in Section IV. A discussion of the experimental applicability of these two problems, the criteria for neglecting viscous effects, and the implications for capillary wave damping are also given.

\section{CAPILLARY WAVES AND SURFACE TENSION DISCONTINUITIES}

\section{A. Formulation}

Consider the propagation of small amplitude capillary waves on a fluid with kinematic viscosity $\nu$, density $\rho$ and of depth $H$ (Figure 1a). A plane wave from far away impinges on a circular domain of radius $a$ which resides on the otherwise homogeneous interface. First, we treat the classical capillary wave problem with surface tension as the restoring force. Second, we study scattering from a bending rigidity discontinuity in Section III. In all cases, gravitational effects on wave propagation are neglected.

For a surface tension discontinuity, the constant surface tension of the interface exterior to the domain is denoted $\sigma_{0}$ and of the domain is denoted $\sigma_{1}$. Mechanical stability of such a circular domain requires that a line tension act along the domain boundary. In monomolecular films, the line tension arises from the van der Waals interactions between the molecules. and line tension effects are higher order since the domain is assumed to deviate only slightly from a circular shape. We thus apply linearized capillary wave theory to obtain the scattering produced by the discontinuous region of surface tension. Both the velocity field and height of the fluctuating interface are calculated. This interface displacement is often measured experimentally by light scattering techniques 13.15

Assuming an irrotational flow, the velocity may be represented by a potential

$$
v_{i}\left(x_{j}, t\right) \equiv \frac{\partial \phi\left(x_{j}, t\right)}{\partial x_{i}}
$$

The flow is assumed to be incompressible so that

$$
\nabla^{2} \phi=0
$$

It is convenient to use cylindrical polar coordinates $(R, \theta, Z)$ and later the dimensionless coordinates $(r, \theta, z)$ are used. For small amplitude disturbances, the momentum equation is linearized, so that the pressure $p=-\rho \partial_{t} \phi$, and the boundary conditions are also linearized and applied at $Z=0$. Finally, the surface height is denoted $\eta(R, \theta, t)$. Effects of viscosity are treated in Appendix A.

The boundary conditions to be applied are a kinematic constraint

$$
\frac{\partial \eta}{\partial t}=\frac{\partial \phi}{\partial Z} \quad \text { at } \quad Z=0
$$


a dynamic condition which follows from the normal stress balance,

$$
\sigma \nabla_{\perp}^{2} \eta=\rho \frac{\partial \phi}{\partial t} \quad \text { at } \quad Z=0
$$

where $\nabla_{\perp}^{2}$ is the two-dimensional Laplacian expressed in the surface coordinates, and an impenetrability condition at the bottom of the trough

$$
\frac{\partial \phi}{\partial Z}=0 \quad \text { at } \quad Z=-H
$$

Taking a time derivative of (2.4) and using the kinematic condition (2.3) yields

$$
\left[\sigma \nabla_{\perp}^{2} \partial_{Z}-\rho \partial_{t}^{2}\right] \phi=0
$$

which, along with $(2.2)$ and $(2.5)$, govern the evolution of the velocity potential.

For a plane wave propagating in a fluid with homogeneous surface properties and depth $H, \phi \propto e^{i k_{0} X-i \omega t} \cosh k(Z+$ $H$ ) determines the well-known dispersion relation for surface capillary waves excited at frequency $\omega$,

$$
\rho \omega^{2}=\sigma k_{0}^{3} \tanh k_{0} H
$$

where $k_{0}=2 \pi / \lambda$ denotes the wavevector and $\lambda$ is the wavelength. The normal stress contribution to (2.4) due to the gravitational acceleration $g$ may be neglected provided

$$
k_{0} \gg\left(\frac{\rho g}{\sigma}\right)^{1 / 2}
$$

Thus, for the air-water interface, gravity can neglected for wavelengths $\lambda=2 \pi / k \ll 1.7 \mathrm{~cm}$.

In most experimental situations, $\omega$ is a real, fixed frequency determined by the wavemaker and equation (2.7) defines the wavevector $k_{0}(\omega)$ as a function of $\omega$ for a particular $\sigma$. When the viscosity of the fluid is explicitly included in the linear analysis of surface fluctuations, the wavevectors have a positive imaginary component 19 (derived in Appendix A) corresponding to viscous damping of the surface fluctuations.

\section{B. Scattering by a circular surface domain}

We now consider the disturbance to the plane progressive wave caused by a circular domain on the interface. This domain changes the surface tension within it such that

$$
\sigma(r)=\sigma_{0} \Theta(R-a)+\sigma_{1} \Theta(-R+a)
$$

where $\Theta(x)$ is the Heaviside function which is unity for $x>0$ and is zero otherwise.

The velocity potential in the lower half-space can written as an incident wave coming from $X \rightarrow-\infty$, plus a scattered velocity potential, $\psi(R, \theta, Z)$, which is assumed to be outgoing and to decay as $R \rightarrow \infty$ :

$$
\phi(R, \theta, Z, t)=\frac{\cosh k_{0}(H+Z)}{\cosh k_{0} H} e^{i k_{0} X-i \omega t}+\psi(R, \theta, Z) e^{-i \omega t},
$$

where $k_{0}$ is given by the dispersion relation (2.7) with $\sigma=\sigma_{0}$. Using the $\theta \rightarrow-\theta$ angular symmetry of the problem, the scattered potential may be expanded as

$$
\psi(R, \theta, Z)=\sum_{m=0}^{\infty} \cos m \theta \int_{0}^{\infty} d k \psi_{m}(k) J_{m}(k R)\left[\frac{\cosh k(H+Z)}{\cosh k H}\right]
$$

where $\theta$ is measured from the incident wavevector (see Figure 1a) and the $J_{m}(k R)$ terms ensure that the potential remains bounded as $R \rightarrow 0$. Equation (2.11) is a complete expansion in Bessel functions and describes the analytic function $\psi(R, \theta, Z)$ obeying the Laplace equation in the domain $z \leq 0$ and satisfying the no-penetration condition at $Z=-H$. Later in the analysis, we will see that the choice of outgoing disturbances from the domain (a radiation condition) requires that the first kind Hankel functions, $H_{m}^{(1)}(k R)$, represent the dominant contribution for large $R$. 
The functions $\psi_{m}(k)$ are determined by first substituting (2.10) into (2.6). Since the incident wave in (2.10) automatically satisfies the normal stress boundary condition at the interface, then outside the circular region $(R>a)$, where the surface tension is $\sigma_{0}$, we have

$$
\left.\left[\sigma_{0} \nabla_{\perp}^{2} \partial_{Z}+\rho \omega^{2}\right] \psi(R, \theta, Z)\right|_{Z=0}=0 \quad(R>a)
$$

while inside the circular domain we have

$$
\left.\left[\sigma_{1} \nabla_{\perp}^{2} \partial_{Z}+\rho \omega^{2}\right] \psi(R, \theta, Z)\right|_{Z=0}=k_{0}^{3}\left(\sigma_{1}-\sigma_{0}\right) e^{i k_{0} R \cos \theta} \tanh k_{0} H \quad(0 \leq R<a) .
$$

We can further expand the right hand side of (2.13) using the identity 20

$$
e^{i k_{0} R \cos \theta}=\left[J_{0}\left(k_{0} R\right)+2 \sum_{p=1}^{\infty} i^{p} J_{p}\left(k_{0} R\right) \cos p \theta\right] .
$$

Substituting (2.14) and (2.11) into (2.13) and multiplying both sides of (2.13) and (2.12) by $\cos n \theta$, then integrating $\theta$ from 0 to $2 \pi$, we obtain a set of dual integral equations for each $n \geq 0$ :

$$
\begin{array}{ll}
\int_{0}^{\infty} d k J_{n}(k R) \psi_{n}(k)\left(\rho \omega^{2}-\sigma_{0} k^{3} \tanh k H\right)=0, & (R>a) \\
\int_{0}^{\infty} d k J_{n}(k R) \psi_{n}(k)\left(\rho \omega^{2}-\sigma_{1} k^{3} \tanh k H\right)= & (0 \leq R<a) . \\
\left(2-\delta_{n, 0}\right) i^{n}\left(\sigma_{1}-\sigma_{0}\right) k_{0}^{3} J_{n}\left(k_{0} R\right) \tanh k_{0} H &
\end{array}
$$

Rescaling $r=R / a, h=H / a, k a=q$, and letting $\Lambda=\sigma_{0} / \sigma_{1}$ denote the surface tension ratio, we can rewrite this system of integral equations as

$$
\begin{array}{ll}
\int_{0}^{\infty} F_{n}(q) J_{n}(q r) d q=0, & (r>1) \\
\int_{0}^{\infty} F_{n}(q) G(q) J_{n}(q r) d q=\Delta_{n} J_{n}\left(q_{0} r\right), & (0 \leq r<1)
\end{array}
$$

where

$$
\begin{gathered}
F_{n}(q) \equiv \frac{\left(q_{0}^{3} \tanh q_{0} h-q^{3} \tanh q h\right)}{i^{n} a} \psi_{n}(q / a) \\
G(q) \equiv \frac{q^{3} \tanh q h-q_{1}^{3} \tanh q_{1} h}{q^{3} \tanh q h-q_{0}^{3} \tanh q_{0} h}
\end{gathered}
$$

and

$$
\Delta_{n} \equiv\left(2-\delta_{n, 0}\right)(1-\Lambda) q_{0}^{3} \tanh q_{0} h
$$

In (2.17), (2.18), and (2.19), we have introduced the dimensionless wavevectors

$$
q_{j}^{3} \tanh q_{j} h \equiv \frac{\rho \omega^{2} a^{3}}{\sigma_{j}} \quad(j=0,1)
$$

We note that $q_{1}=k_{1} a$ denotes the dimensionless wavevector which would be measured if the entire interface were homogeneous with surface tension $\sigma_{1}$.

Finally, as mentioned above, the wavevectors can be interpreted as having a small positive imaginary part which ensures that the potentials vanish as $R \rightarrow \infty$. This behavior can be attributed to the effects of small viscous damping. The small damping may be explicitly accounted for by studying the viscous flow problem and taking the limit $\nu \rightarrow 0^{+}$. A dual integral equation formulation to the viscous flow problem for finite $\nu$ is given for completeness in Appendix A. Here, we treat fully the inviscid problem, but simply allow the wavevectors to have an infinitesimally small positive imaginary part as is common in scattering calculations.21 


\section{Solution of the integral equations}

The flow problem contains three dimensionless parameters: $q_{0}$, the incident wavevector; $h$, the fluid depth; and $\Lambda$, the surface tension ratio characterizing the surface discontinuity. It is also clear from $(2.19)$ that $\Lambda=1$ gives $\Delta_{n}=0$ for all $n \geq 0$ so that using (2.16) and (2.17) $\psi_{n}=0$ and hence there is no scattering.

The solution of the dual integral equations (2.16) for the unknown functions $F_{n}(q)$ can be readily determined numerically using a procedure introduced by Tranter 22 which we describe for completeness. Begin by noticing the form of the Weber-Schafheitlin discontinuous integral23

$$
\int_{0}^{\infty} q^{1-\beta} J_{2 m+n+\beta}(q) J_{n}(q r) d q= \begin{cases}\frac{\Gamma(n+m+1) r^{n}\left(1-r^{2}\right)^{\beta-1}}{2^{\beta-1} \Gamma(n+1) \Gamma(m+\beta)} \mathcal{F}_{m}\left(\beta+n, n+1 ; r^{2}\right) & (0 \leq r<1) \\ 0 & (r>1)\end{cases}
$$

where $\mathcal{F}_{m}\left(\beta+n, n+1 ; r^{2}\right)$ is the Jacobi polynomial of order $m$.20 Based upon the analogy of the right-hand-side of (2.21) and (2.16) for $r>1$, it is natural to expand the unknown functions $F_{n}(q)$ in terms of Bessel functions as

$$
F_{n}(q) \equiv q^{1-\beta} \sum_{m=0}^{\infty} A_{m}^{(n)} J_{2 m+n+\beta}(q),
$$

where $\beta$ is a constant which may be chosen later to improve the rates of convergence of the required numerical integrations. With this representation of $F_{n}(q)$, the first equation in 2.16) is automatically satisfied. Substituting (2.22) into the second equation in 2.16$)$, we are left with

$$
\sum_{m=0}^{\infty} A_{m}^{(n)} \int_{0}^{\infty} d q q^{1-\beta} J_{2 m+n+\beta}(q) J_{n}(q r) G(q)=\Delta_{n} J_{n}\left(q_{0} r\right) \quad 0 \leq r<1 .
$$

Multiplying both sides of $(2.23)$ by $r^{n+1}\left(1-r^{2}\right)^{\beta-1} \mathcal{F}_{l}\left(\beta+n, n+1 ; r^{2}\right)$ and integrating $r$ from 0 to 1 gives for each $n$, where $n=0,1,2,3 \ldots$, a system of equations

$$
\sum_{m=0}^{\infty} A_{m}^{(n)} \int_{0}^{\infty} d q q^{1-2 \beta} J_{2 m+n+\beta}(q) J_{2 l+n+\beta}(q) G(q)=q_{0}^{-\beta} \Delta_{n} J_{2 l+n+\beta}\left(q_{0}\right) \quad l=0,1, \ldots
$$

Equation (2.23) represents an infinite matrix for the coefficients $A_{m}^{(n)}$ and can be written succinctly as

$$
\sum_{m=0}^{\infty} A_{m}^{(n)} L_{m l}^{(n)}\left(\beta ; q_{0}, \Lambda\right)=q_{0}^{2 \beta-2} \Delta_{n} J_{2 l+n+\beta}\left(q_{0}\right) \quad(n, l \geq 0)
$$

where

$$
\begin{aligned}
L_{m l}^{(n)}\left(\beta ; q_{0}, \Lambda\right)= & \int_{0}^{\infty} \frac{d x}{x^{2 \beta-1}} \frac{\left(x^{3} \tanh q_{0} h x-\Lambda \tanh q_{0} h\right)}{\left(x^{3} \tanh q_{0} h x-\tanh q_{0} h\right)} J_{2 m+n+\beta}\left(q_{0} x\right) J_{2 l+n+\beta}\left(q_{0} x\right) \\
& \equiv \int_{0}^{\infty} d x I_{m l}^{(n)}\left(q_{0}, h, \Lambda ; x\right)
\end{aligned}
$$

The infinite set of linear equations (2.25) determines the coefficients of the expansion of the functions $F_{n}(q)$ from which the Fourier coefficients $\psi_{n}(k)$ are known by (2.17) and hence the scattered wave $\psi(r, \theta, z)$ is determined.

The integral in equation 2.26 has a pole on the real axis at $x=1$ (i.e. $q=q_{0}$ ). We may treat this pole in a manner consistent with outgoing waves 21 by interpreting the pole as having a positive imaginary part, $\operatorname{Im}\left\{q_{j}\right\}=\epsilon>0$ and taking the limit $\epsilon \rightarrow 0$. This limit is consistent with viscous effects becoming small, $\nu \rightarrow 0^{+}$. In the limit $\epsilon \rightarrow 0$, the integral can be evaluated by taking the path of integration through a small semicircle below the pole at $x=1$ which yields

$$
\begin{aligned}
L_{m l}^{(n)}=i \pi(1- & \Lambda)\left[\frac{\sinh q_{0} h \cosh q_{0} h}{3 \sinh q_{0} h \cosh q_{0} h+q_{0} h}\right] J_{2 m+n+\beta}\left(q_{0}\right) J_{2 l+n+\beta}\left(q_{0}\right) \\
& +\int_{0}^{1} d x\left[I_{m l}^{(n)}\left(q_{0}, h, \Lambda ; x\right)+I_{m l}^{(n)}\left(q_{0}, h, \Lambda ; 2-x\right)\right]+\int_{2}^{\infty} d x I_{m l}^{(n)}\left(q_{0}, h, \Lambda ; x\right)
\end{aligned}
$$


where we have separated the integrable real part of the integral over $I_{m l}^{(n)}$ from the contribution from the semicircle below the pole. The remaining integrals are evaluated numerically.

We next solve a truncated system of linear equations (2.25) and so obtain the $A_{m}^{(n)}$ 's which depend on $q_{0}, h$, and $\Lambda$. The scattered potential follows from

$$
\psi(r, \theta, z ; h)=\sum_{n, m=0}^{\infty} i^{n} A_{m}^{(n)} \int_{0}^{\infty} d q \frac{q^{1-\beta} \cos n \theta}{q_{0}^{3} \tanh q_{0} h-q^{3} \tanh q h} \frac{\cosh q(h+z)}{\cosh q h} J_{2 m+n+\beta}(q) J_{n}(q r)
$$

from which we find the interfacial height displacement of the scattered part of the wave

$$
\eta_{s}(r, \theta)=\left.\frac{i}{\omega} \frac{\partial}{\partial z} \psi(r, \theta, z)\right|_{z=0}=\frac{i}{\omega} \sum_{n, m=0}^{\infty} i^{n} A_{m}^{(n)} \int_{0}^{\infty} d q \frac{q^{2-\beta} \cos n \theta}{q_{0}^{3} \tanh q_{0} h-q^{3} \tanh q h} J_{2 m+n+\beta}(q) J_{n}(q r) .
$$

Equations (2.28) and (2.29) also have poles on the real axis at $q=q_{0}$, which we choose to integrate using a semicircle contour below the pole. This treatment fixes the scattered waves as outgoing. In particular, to evaluate the integral in (2.28), we replace the $J_{n}(q r)$ term with $\frac{1}{2}\left(H_{n}^{(1)}(q r)+H_{n}^{(2)}(q r)\right)$. For $r>1$, the integral over $J_{2 m+n+\beta}(q) H_{n}^{(1)}(q r)$ is closed by the contour in the upper half-plane shown in Figure 2. The detour in quadrant one corresponds to a branch cut at $q=\sqrt{i \omega a^{2} / \nu}$ and is derived in Appendix A. Similarly, the $J_{2 m+n+\beta}(q) H_{n}^{(2)}(q r)$ integral is closed in the lower half-plane. Only the $H_{n}^{(1)}$ integral encloses the pole at $q=q_{0}$. Thus, only the outgoing waves proportional to $H_{n}^{(1)}\left(q_{0} r\right)$ are important for large $r$. The final result can be expressed in the form

$$
\begin{array}{r}
\int_{0}^{\infty} d q \frac{q^{1-\beta}}{q_{0}^{3} \tanh q_{0} h-q^{3} \tanh q h} \frac{\cosh q_{0}(h+z)}{\cosh q_{0} h} J_{2 m+n+\beta}(q) J_{n}(q r) \simeq \\
-\pi i \frac{\cosh q_{0} h \cosh q_{0}(h+z)}{q_{0}^{\beta+1}\left(3 \sinh q_{0} h \cosh q_{0} h+q_{0} h\right)} J_{2 m+n+\beta}\left(q_{0}\right) H_{n}^{(1)}\left(q_{0} r\right)-\Gamma_{+}-\Gamma_{-} \quad(r>1)
\end{array}
$$

The contributions to the integral from the integrations along the imaginary axis in Figure $2, \Gamma_{ \pm}$, can be shown to be negligible compared to $H_{n}^{(1)}\left(q_{0} r\right)$ as $r \rightarrow \infty$. Asymptotically,

$$
H_{n}^{(1)}\left(q_{0} r\right) \simeq \sqrt{\frac{2}{\pi q_{0} r}} e^{i q_{0} r-i \pi n / 2-i \pi / 4} \quad(r \rightarrow \infty)
$$

which leads us to write the far-field potential in the form

$$
\psi(r, \theta, z ; h) \simeq f(\theta ; \Lambda, h) \frac{e^{i q_{0} r}}{\sqrt{r}} \frac{\cosh q_{0}(h+z)}{\cosh q_{0} h} \quad(r \rightarrow \infty)
$$

where the scattering amplitude is obtained by combining (2.28) and (2.30):

$$
f(\theta ; \Lambda, h) \equiv \frac{e^{-3 \pi i / 4}}{q_{0}^{\beta+1}} \sqrt{\frac{2 \pi}{q_{0}}}\left[\frac{\cosh ^{2} q_{0} h}{3 \sinh q_{0} h \cosh q_{0} h+q_{0} h}\right] \sum_{n, m=0}^{\infty} A_{m}^{(n)} J_{2 m+n+\beta}\left(q_{0}\right) \cos n \theta .
$$

The total scattering section is denoted $\Sigma$ and is given by

$$
\Sigma\left(q_{0}, \Lambda, h\right) \equiv \int_{0}^{2 \pi} d \theta|f(\theta)|^{2}=\frac{2 \pi^{2}}{q_{0}^{2 \beta+3}}\left[\frac{\cosh ^{2} q_{0} h}{3 \sinh q_{0} h \cosh q_{0} h+q_{0} h}\right]^{2} \sum_{n=0}^{\infty}\left|\sum_{m=0}^{\infty} A_{m}^{(n)} J_{2 m+n+\beta}\left(q_{0}\right)\right|^{2},
$$

where $\sum^{\prime}$ denotes multiplying by 2 for the $n=0$ term. Equations (2.33) and (2.34) are the principle results of this section. 


\section{Numerical Evaluation of $A_{m}^{(n)}$}

The linear set of equations 2.25) was solved by numerically integrating the functions in (2.27) to obtain complex matrix coefficients and then, for each $n$, an $M \times M$ linear system was solved to determine $A_{m}^{(n)}$. All numerical integrations demanded relative error $10^{-8}$. The dependence of the $A_{m}^{(n)}$, s on the matrix size was found to be negligible $\left(<10^{-6}\right.$ ) provided $M \geq 14$. $\beta$ was chosen to guarantee convergence of the integrals and we chose $\beta=1$ for our calculations as this appeared to give the fastest convergence for large values of $m, l$. A numerical integration technique for infinite integrals involving products of Bessel functions, developed recently by Lucas, 24 was used for the integrations of the kernels in (2.27).

\section{E. Wave scattering on a heterogeneous free membrane}

Since we are not aware of an analogy to capillary wave scattering from a boundary condition inhomogeneity as studied above, it is useful to consider a related scattering problem with a similar geometry. In particular, we treat wave propagation on a stretched elastic membrane and calculate the scattering due to a circular region with tension $T_{1}$ which is imbedded in a two-dimensional membrane with tension $T_{0}$. Vertical displacements of this membrane obey the usual wave equation. In Section IV the scattering from this model is compared with that derived in the previous section.

The configuration described in this section can in principle be realized experimentally by embedding a thin flexible loop of thread in a soap film. Surfactant can then be added to the interior of the loop to decrease the surface tension within the circle. The thread now supplies the line tension $\gamma$, which acts in the tangent plane of the interface and satisfies

$$
\frac{\gamma}{a}=\left(T_{0}-T_{1}\right)
$$

The effects of an underlying liquid substrate are not present in this example and the only dynamical variable of the membrane is the height fluctuation $\eta(r, \theta, t)$ which obeys a wave equation $\partial_{t}^{2} \eta=c^{2} \nabla_{\perp}^{2} \eta$ with a dispersion relation

$$
q_{j}^{2}=\frac{\omega^{2} a^{2}}{c_{j}^{2}} \quad(j=0,1)
$$

where we have used the same rescaled dimensionless quantities as in the previous section (e. g., $q=k a$ ). The wave speeds for the different regions are $c_{j}=\sqrt{T_{j} / \rho_{m}}$ where $\rho_{m}$ denotes the areal mass density of the thin film.

We now wish to consider scattering of an incident plane wave, $e^{i q_{0} r \cos \theta-i \omega t}$, by this circular region of different tension. The membrane displacement, $\eta e^{-i \omega t}$, outside the circular region takes the form

$$
\eta_{\text {out }}(r, \theta)=e^{i q_{0} r \cos \theta}+\eta_{s}(r, \theta) \quad(r>1)
$$

where the outgoing scattered height can be expanded as

$$
\eta_{s}(r, \theta)=\sum_{n=0}^{\infty} A_{n} H_{n}^{(1)}\left(q_{0} r\right) \cos n \theta . \quad(r>1)
$$

Similarly, the membrane displacement inside the circle can be expanded as

$$
\eta_{\text {in }}(r, \theta)=\sum_{n=0}^{\infty} B_{n} J_{n}\left(q_{1} r\right) \cos n \theta . \quad(0 \leq r<1)
$$

At the domain boundary $(r=1)$, continuity of the sheet requires

$$
\eta_{\text {out }}(1, \theta)=\eta_{\text {in }}(1, \theta)
$$

which also implies $\partial_{\theta} \eta$ is continuous across $r=1$. The second boundary condition requires careful consideration of the directionality of the line tension. Since both the interfacial tensions $T_{j}$ and the line tension $\gamma$ act in the surface of the membrane, a balance of forces in the $z$ direction requires continuity of slopes

$$
\partial_{r} \eta_{\text {out }}(1, \theta)=\partial_{r} \eta_{\text {in }}(1, \theta)
$$


Substituting the expressions for the height displacements inside and outside the loop into the two conditions (2.40) and (2.41) yields

$$
A_{n}=\left(2-\delta_{n, 0}\right) i^{n} \frac{q_{0} J_{n}\left(q_{1}\right)\left[J_{n+1}\left(q_{0}\right)-J_{n-1}\left(q_{0}\right)\right]-q_{1} J_{n}\left(q_{0}\right)\left[J_{n+1}\left(q_{1}\right)-J_{n-1}\left(q_{1}\right)\right]}{q_{1} H_{n}^{(1)}\left(q_{0}\right)\left[J_{n+1}\left(q_{1}\right)-J_{n-1}\left(q_{1}\right)\right]-q_{0} J_{n}\left(q_{1}\right)\left[H_{n+1}^{(1)}\left(q_{0}\right)-H_{n-1}^{(1)}\left(q_{0}\right)\right]},
$$

for $n \geq 0$. We note that the tension ratio enters this result since $q_{1} / q_{0}=\sqrt{T_{0} / T_{1}}$. At large distances, $r \rightarrow \infty$, the membrane displacement (2.37) is

$$
\eta_{\text {out }}(r, \theta) \simeq e^{i q_{0} r \cos \theta}+f(\theta) \frac{e^{i q_{0} r}}{\sqrt{r}}
$$

with

$$
f\left(\theta ; T_{0} / T_{1}\right)=\sqrt{\frac{2}{\pi q_{0}}} e^{-i \pi / 4} \sum_{n=0}^{\infty}(-i)^{n} A_{n} \cos n \theta .
$$

The scattering cross section for this process is given by

$$
\Sigma\left(q_{0}, T_{0} / T_{1}\right)=\frac{4}{q_{0}}\left|A_{0}\right|^{2}+\frac{2}{q_{0}} \sum_{n=1}^{\infty}\left|A_{n}\right|^{2}
$$

which will be compared in Section IV with the corresponding results obtained in IIC.

\section{SCATTERING WITH BENDING RIGIDITY DISCONTINUITIES}

\section{A. Waves on a fluid coupled membrane with bending rigidity}

In this section, we consider the case of a thin elastic plate overlying a fluid whose motion is assumed irrotational and satisfies (2.2). Imbedded in the plate is a circular region with different elastic constants. This circular domain thus acts to scatter an incoming transverse (bending) vibration. Physical manifestations of this problem are membranes with phase separated domains, with imbedded impurities or defects, or other films with a patch of different flexural rigidity 25 (see Figure 1b).

We assume that the only normal restoring force at the interface is an elastic resistance to bending which modifies the boundary conditions (2.12) and (2.13). Balancing the pressure, $p=-\rho \partial_{t} \phi$, the bending forces, and the inertia of the membrane with mass density $\rho_{p}(2.6)$, the equation for the plate displacement $\zeta$ is 26

$$
\left[D \nabla_{\perp}^{4}+\rho_{p} \partial_{t}^{2}\right] \zeta(R, \theta)=p .
$$

Here $D$ is the bending rigidity, which for an isotropic harmonic solid is typically defined as 22

$$
D_{j}=\frac{E_{j} d^{3}}{12\left(1-\mu_{j}^{2}\right)}
$$

where $d$ is the plate thickness, and $E_{j}$ and $\mu_{j}$ are Young's moduli and Poisson's ratios, respectively. The elastic parameters will be assumed to have different, though uniform, values inside and outside the circular domain. In microscopic systems such as monolayers and bilayers where the "plate" thickness has a molecular scale, the bending rigidity is typically denoted $\kappa$, but the physical interpretation of $\kappa$ in terms of material parameters as given by (3.2) is not valid.

Taking a time derivative and using the kinematic constraint (2.3), we obtain the dynamic boundary condition

$$
\left.\left[D \nabla_{\perp}^{4} \partial_{Z}+\left(\rho+\rho_{p} \partial_{Z}\right) \partial_{t}^{2}\right] \phi(R, \theta, Z)\right|_{Z=0}=0 .
$$

The solution of this problem is similar to that of the surface tension discontinuity. However, rather than considering the general case of arbitrary values of fluid density and membrane density, we simplify the problem and consider two limiting, important cases. The first case is when the normal forces from fluid pressure dominate the plate's own inertial response. The second situation, discussed in subsection B, neglects the effects of the underlying fluid. We 
note an important difference between these two cases. In the formulation for the fluid coupled, massless plate, no further conditions on the behavior of the fields at $r=1, z=0$ can be imposed. Here, fluid velocities, surface displacements, and their derivatives are continuous. However, the physical model considered may require extra conditions at $r=1, z=0$ which cannot be satisfied by the velocity potential of the irrotational fluid. These extra boundary conditions are part of plate theory and are incorporated in the second case, the scattering of free plate vibrations.

When the plate mass is neglected, the dispersion relation derived from (3.1) is

$$
q_{j}^{5} \tanh q_{j} h=\frac{\rho \omega^{2} a^{5}}{D_{j}} .
$$

Equation (3.4) is the massless plate limit, $\rho_{p} / \lambda \ll \rho$, which, upon solving for $\lambda=2 \pi / k$ using (3.4) for $h=\infty$, is consistent with

$$
\rho_{p} \ll\left(\frac{\rho^{4} D}{\omega^{2} a}\right)^{1 / 5} .
$$

The detailed scattering calculation has the identical form to that of the surface tension discontinuity problem except that the functions $F(q)$ and $G(q)$ are changed and the coefficients 2.26) are modified. It is straightforward to obtain

$$
\begin{aligned}
L_{m l}^{(n)}\left(q_{0}, h, \Lambda_{d} ; x\right) & =i \pi\left(1-\Lambda_{d}\right)\left[\frac{\sinh q_{0} h \cosh q_{0} h}{5 \sinh q_{0} h \cosh q_{0} h+q_{0} h}\right] J_{2 m+n+\beta}\left(q_{0}\right) J_{2 l+n+\beta}\left(q_{0}\right) \\
& +\int_{0}^{1} d x\left[I_{m l}^{(n)}\left(q_{0}, h, \Lambda_{d} ; x\right)+I_{m l}^{(n)}\left(q_{0}, h, \Lambda_{d} ; 2-x\right)\right]+\int_{2}^{\infty} d x I_{m l}^{(n)}\left(q_{0}, h, \Lambda_{d} ; x\right)
\end{aligned}
$$

with

$$
I_{m l}^{(n)}\left(q_{0}, h, \Lambda ; x\right) \equiv x^{1-2 \beta} \frac{x^{5} \tanh q_{0} h x-\Lambda_{d} \tanh q_{0} h}{x^{5} \tanh q_{0} h x-\tanh q_{0} h}
$$

and $\Lambda_{d} \equiv D_{0} / D_{1}$ denotes the ratio of outer to inner bending rigidities. The scattering amplitude $f$ and total scattering cross section $\Sigma$ then follow from (2.33) and (2.34) by solving for the necessary coefficients using (2.25), (with $\Lambda_{d}$ replacing $\Lambda$ ), and (3.6).

\section{B. Bending waves of a free plate}

Here it is instructive to study scattering in the uncoupled plate limit. Provided $a \rho_{p} \omega^{2 / 5} \gg \rho^{4 / 5} D^{1 / 5}$, the pressure from the underlying fluid can be neglected in the force balance (3.1) and it is no longer necessary to consider the underlying fluid (and so the solution for the velocity potential). Setting $p=0$ in (3.1) yields a wave equation for $\zeta$ with the dispersion relation

$$
q_{j}^{4}=\frac{\rho_{p} d \omega^{2} a^{4}}{D_{j}} \quad j=0,1
$$

for waves outside $(j=0)$ and inside $(j=1)$ the domain. We treat the dynamics in a manner typically used in plate theory, where shear stresses and bending moments within the plate are balanced.27

As in Section IIE, the solution for $\zeta$ outside and inside the circular domain can be expressed as

$$
\zeta_{\text {out }}=e^{i q_{0} r \cos \theta-i \omega t}+\zeta_{s}(r, \theta) e^{-i \omega t} \quad(r>1)
$$

where

$$
\zeta_{s}(r, \theta)=\sum_{n=0}^{\infty}\left[A_{n} H_{n}^{(1)}\left(q_{0} r\right)+B_{n} K_{n}\left(q_{0} r\right)\right] \cos n \theta \quad(r>1)
$$

and

$$
\zeta_{\text {in }}(r, \theta)=\sum_{n=0}^{\infty}\left[C_{n} J_{n}\left(q_{1} r\right)+E_{n} I_{n}\left(q_{1} r\right)\right] \cos n \theta \quad(0 \leq r<1)
$$


To determine the coefficients $A_{n}, B_{n}, C_{n}$, and $E_{n}$, we apply four constraints at the boundary $r=1$ : continuity of the plate displacements, the pure bending approximation, balance of vertical shear forces, and balance of rotational moments. These quantities can be expressed in the following terms: $\zeta_{i n}^{-}=\zeta_{o u t}^{+}, \beta_{r}^{-}=\beta_{r}^{+}, M_{r r}^{-}=M_{r r}^{+}$, and $V_{r z}^{-}=$ $V_{r z}^{+}$, where $\beta_{r}, M_{r r}$, and $V_{r z}$ are the bending angle, the bending moment, and the Kirchhoff shear stress resultant respectively, and \pm denote the functions evaluated as $r \rightarrow 1$ from outside and inside, respectively.26.27 Expressing these four conditions in terms of the out-of-plane displacements, we conclude that

$$
\zeta, \quad \frac{\partial \zeta}{\partial r}, \quad D(r)\left[\nabla^{2} \zeta+(\mu(r)-1)\left(\frac{1}{r} \frac{\partial \zeta}{\partial r}+\frac{1}{r^{2}} \frac{\partial^{2} \zeta}{\partial \theta^{2}}\right)\right]
$$

and

$$
D(r)\left[\nabla^{2} \frac{\partial \zeta}{\partial r}+\frac{\mu(r)-1}{r^{2}}\left(\frac{1}{r} \frac{\partial^{2} \zeta}{\partial \theta^{2}}-\frac{\partial^{3} \zeta}{\partial \theta^{2} \partial r}\right)\right]
$$

are all continuous across the boundary of the circular inclusion. The latter two boundary conditions at the domain boundary are not enforced for the fluid coupled problem, as discussed in the next section. Substituting the expansions (3.9 3.11) into these four boundary conditions yields a linear set of equations which determine the unknown coefficients. In particular, we find

$$
A_{n}=\frac{\operatorname{det} \mathbf{N}}{\operatorname{det} \mathbf{M}}
$$

where the matrices $\mathbf{M}$ and $\mathbf{N}$ are given in Appendix B. Expressions for the scattering amplitude and total scattering cross section, which are functions of the $A_{n}$, are identical to those of the tension discontinuity problem and are given by equations (2.44) and (2.45).

\section{DISCUSSION}

\section{A. Results}

We first examine capillary wave scattering on a heterogeneous surface using equations $(2.33)$ and $(2.34)$, and compare with scattering in a free membrane, equation (2.45). Polar plots of the scattering amplitude, $|f(\theta, h=\infty)|$, for various incoming wavevectors $q_{0}$ and $\Lambda=2$ are shown in Figure 3. In the long wavelength limit, $q_{0} \ll 1$, the domain is nearly a point scatterer, and the scattering is nearly isotropic as expected; even for $q_{0} \approx 1$, there is almost no variation with $\theta$. Distinct lobe structures develop when the incoming wavelength is about three times the radius of the scattering domain. Figures 4a-d illustrate, respectively, the effect of subphase depth on scattering for wavevectors $q_{0}=1.0,2.0,3.0$, and 4.0, again for $\Lambda=2$. In each case, depths $h=0.1,0.5$, and $h=\infty$ are considered. For these wavevectors, decreasing $h$ decreases the scattering amplitude for all $\theta$, but has almost no effect on the angular variations.

The total scattering cross sections as a function of the reduced incident wavevector for $\Lambda=2.0$ and 10.0 are shown for various depths in Figures 5a,b respectively. The depth dependence of $\Sigma$ is shown in Figure 6. Figures 5ab show oscillations in $\Sigma$ as $q_{0}$ is varied. Unlike examples of sound wave scattering from an infinitely rigid obstacle, 28 where a monotonic variation of $\Sigma$ with $q_{0}$ is expected, the calculations presented here exhibit a different and more complex dynamical behavior 29 The curves for $\Sigma$ have oscillations and maxima analogous to Mie resonances in the scattering of radiation from a dielectric sphere 30 In Figure 6, a weak variation of $\Sigma$ with $h$ is observed which varies most significantly for intermediate $q_{0}$. We note that for shallow underlying fluids viscous dissipation is important when $\nu \gg a^{2} \omega h^{2}$, and is discussed further below. We have also compared our results with the asymptotic expression in the small wavenumber limit $\left(q_{0} \ll 1\right), \Sigma=\frac{\pi^{2}}{9}(\Lambda-1)^{2} q^{3}$, developed by Chou and Nelson.16 The agreement is good as displayed in Figure 5c.

We compare the above results with those of scattering in a free membrane (the effects of the underlying fluid are neglected, section IIID), shown as the darker dashed curves in Figures 5a,b. Although these two problems are geometrically similar, they have different dispersion relations, so in order to provide a meaningful comparison, we study two cases with the ratio of wavevectors $q_{1} / q_{0}=2^{1 / 3}$ and $10^{1 / 3}\left(\Lambda=\sigma_{0} / \sigma_{1}=2,10\right.$ but $\left.T_{0} / T_{1}=2^{2 / 3}, 10^{2 / 3}\right)$. A plot of (2.45) for $T_{0} / T_{1}=2$ would correspond to a scale change (compression) along the $q_{0}$ axis in Figure 5 . The dependence of $\Sigma$ on $q_{0}$ for scattering in the uncoupled membrane versus the capillary wave problem is qualitatively similar at the same ratio of inner to outer wavevectors. In fact, the scattering cross section for $\Lambda=2.0, h=\infty$ in Figure $5 \mathrm{a}$ is nearly indistinguishable from that of the free membrane. The corresponding amplitudes $|f(\theta, h)|$ for 
the uncoupled membrane, equation (2.44), are nearly identical to the polar plots shown in Figure 4 corresponding to $\Lambda=2.0$. For $\Lambda=10.0$, Figure $5 \mathrm{~b}$, the free membrane model is now clearly distinguishable from the capillary wave scattering case, even after the wavevectors are rescaled to account for the different dispersion relations between a free membrane and a capillary wave.

To illustrate the effects of varying the ratios of the surface parameters, we have plotted in Figure $7 \Sigma\left(q_{0}, \Lambda, h=\infty\right)$ as a function of $\Lambda^{-1}$ for $q_{0}=1.0,2.0$, and 3.0. The limit $\Lambda^{-1}=\sigma_{1} / \sigma_{0} \rightarrow \infty$ is associated with an infinitely "hard" domain at the interface which resists increases in surface area, though we note that such monolayer systems are unstable and can exist only in the presence of an externally applied force. Increasing $\Lambda$ from unity corresponds to a more flexible domain, which actively supports internal fluctuations, and leads to a more complicated scattering and far-field disturbance.

We now present results for scattering in interfaces characterized by bending rigidity rather than surface tension. Scattering amplitudes for the fluid-coupled membrane for $q_{1} / q_{0}=2^{1 / 3}$ (corresponding to $D_{0} / D_{1}=2^{5 / 3}$ ) are shown in Figures $8 \mathrm{a}$-d for $q_{0}=1.0,2.0,3.0$, and 4.0 and different fluid depths. Total scattering cross sections as a function of $q_{0}$ are plotted in Figure 9. For comparison, the scattering amplitude and cross sections for bending waves of a free plate (no underlying fluid) are depicted by the dotted curves in both Figures 8 and 9. To maintain scale similarity in the internal to external wavelength ratio, $q_{1} / q_{0}=2^{1 / 3}$, we choose $D_{0} / D_{1}=2^{4 / 3}$. In addition, due to the elastic boundary conditions at $r=1$ in the plate model, we need to specify the contrast in both-bending rigidity $D$ and Poisson's ratio $\mu$. The dotted curve in Figure 9 was calculated with $\mu_{0}=0.25, \mu_{1}=-0.2531$ From other numerical simulations, we have found that the cross sections $\Sigma$ are rather insensitive to the discontinuity in Poisson's ratios except for small variations in the fine structure.

The discontinuities present in the higher derivative boundary conditions $(3.12)$ and $(3.13)$ for the free elastic plate make this problem mathematically different from that of the massless bending interface coupled to an underlying fluid, where the velocity potential is assumed harmonic. For the fluid coupled irrotational flow problem, only two boundary conditions at $r=1$ can be satisfied. Solution of the full boundary value problem with four boundary conditions applied at the domain boundary, (3.12) and (3.13), requires consideration of viscous effects in the underlying fluid. The discontinuities at $r=1$ suggested by (3.12) and (3.13) are smoothed out in the thin boundary layer with thickness $\sqrt{\nu / \omega}$ adjacent to the upper surface. It appears that the differences in Figures 8 and 9 for wave scattering along the heterogeneous plate as compared with the comparable capillary wave scattering problem are the discontinuous boundary conditions at $r=1$. In the surface tension problems solved in Section II, the membrane displacement and its first derivative are both continuous and so both (2.40) and (2.41) are satisfied.

The oscillations in $\Sigma\left(q_{0}\right)$ for surface tension scattering, bending rigidity scattering, and scattering in an uncoupled membrane or plate, (Figmes 5 and 9), are similar to those of the cross section of electromagnetic radiation scattering from a dielectric sphere. 30 In that case the radiation field penetrates a sphere with finite dielectric constant; internal reflections constructively or destructively interfere depending on the incoming wavelength. For the problem studied here, capillary wave scattering from an interfacial inhomogeneity, internal waves are supported, and interferences lead to the oscillations in $\Sigma\left(q_{0}\right)$.

\section{B. Experimental Consequences}

Our analysis has been confined to wave scattering from an isolated domain. However, under most experimental conditions the morphology of the monolayer film cannot be directly controlled. For example, when amphiphilic molecules are deposited at the liquid-air interface they form a film which is in a phase separated state consisting of 3 collection of scatterers of different sizes. The density and sizes of these domains depend on surfactant concentration. 0.2 Interfacial surfactant morphology thus affects capillary wave propagation with scattering as a source of enhanced wave attentuation. 111

Experiments on capillary wave damping 1245 show at least one maximum in the attenuation coefficient as a function of total surface concentration of surfactant. Wave amplitudes measured by $|\eta|^{2}$, can be related to the scattering in the dilute scatterer limit (single scattering only) by Beer's law 32

$$
|\eta(x)|^{2} \propto e^{-c \Sigma\left(q_{0}\right) x}
$$

where $c$ is the area concentration of domains of radius $a$. Since the calculated cross section $\Sigma\left(q_{0}\right)$ contains peaks (Fig. 5a,b and Fig. 9) 4,1 at least qualitatively accounts for the maxima in the damping coefficients which are experimentally measured.12 15 Equation (4.1) suggests that peaks may observed as $q_{0}$ is varied. Indeed, the variation in damping as domain size and incoming wavevector are tuned has been qualitatively observed in the experiment of Wang et. al 11 who measured a sharp increase in damping as the incoming wavelength was decreased from $\lambda \gg a$ to $\lambda \approx a$. 
We conclude the discussion by examining the region of validity of the analytical results derived in Sections II and III. Throughout the analysis, effects of viscosity have been neglected. This approximation is valid provided the domain is larger than the viscous length, $\sqrt{\nu / \omega}$, i.e., the Stokes layer adjacent to the fluctuating boundary. Thus we require

$$
\begin{aligned}
& a \gg \sqrt{\frac{\nu}{\omega}} \\
& H \gg \sqrt{\frac{\nu}{\omega}} .
\end{aligned}
$$

When the dispersion relation (2.7) for infinite depth is used to eliminate $\omega$ from (4.3), and a condition that the waves are not too heavily damped by viscosity is imposed, $k_{j}^{2} \ll \omega / \nu$, we obtain validity criteria for $q_{j}=k_{j} a$,

$$
\left(\frac{a^{2} \omega}{\nu}\right)^{1 / 2} \gg q_{j} \gg\left(\frac{\nu^{2} \rho}{a \sigma_{j}}\right)^{1 / 3} \text {. }
$$

For an air-water interface, $\sigma \approx 70$ dynes $/ \mathrm{cm}, \nu \approx 0.01 \mathrm{~cm}^{2} / \mathrm{sec}$, and $\rho \approx 1 \mathrm{gm} / \mathrm{cm}^{3}$. The first relation in $(4.4)$ provides an upper bound of $k_{j}=q_{j} / a \ll \sigma / \rho \nu^{2} \approx 7 \times 10^{5} \mathrm{~cm}^{-1}$ and a lower bound of $k_{j}=q_{j} / a \gg 113 \mathrm{~cm}^{-1}, 5.2 \mathrm{~cm}-1$, and $0.24 \mathrm{~cm}^{-1}$ for domain radii of $a \approx 10 \mu \mathrm{m}, 100 \mu \mathrm{m}$, and $1 \mathrm{~mm}$ respectively.

For a rigid membrane described by the dispersion relation (3.4), the conditions $k_{j}^{2} \ll \omega / \nu$ and 4.2 lead to

$$
k_{j} a=q_{j} \gg \max \left[\left(\frac{a \nu^{2} \rho}{D_{j}}\right)^{1 / 5}, \frac{a \nu^{2} \rho}{D_{j}},\left(\frac{g \rho}{D_{j}}\right)^{1 / 4}\right]
$$

where the last term is a condition due to the neglect of gravity waves. For typical estimates of the bending rigidity in cell membranes, $33 \sim 10^{-12}$ ergs, the above condition (4.5) requires exceedingly small wavelengths, $\lambda \ll 1$ nm, to be satisfied. As an estimate of the range of validity, consider a system with $D=0.1$ ergs. For domain radii of $10 \mu$ m, $100 \mu \mathrm{m}$, and $1 \mathrm{~mm}$, (4.5) requires $k_{j} \gg 63 \mathrm{~cm}^{-1}, 10 \mathrm{~cm}^{-1}$, and $9.95 \mathrm{~cm}^{-1}$, respectively.

\section{SUMMARY}

In this paper, we have formulated an analytical method for studying surface wave scattering due to a circular inhomogeneity in the boundary conditions. The results of the calculations imply that scattering of capillary waves from a circular domain where there is a discontinuity in surface properties (surface tension or bending rigidity) is not very sensitive to the depth of the underlying liquid (see Fig. 7). The scattering patterns $|f(\theta ; h)|$ have lobed structures indicative of angular variations in the far-field disturbance. In addition, an examination of the above results (Figures 5a and 9) show that differences between scattering from a surface tension contrast and a bending rigidity contrast are small if the wavevector ratio, $q_{0} / q_{1}$, of the two problems is maintained constant. For a thin elastic plate, the scattering from a domain of different rigidity has some qualitative differences when compared with the scattering from the fluid coupled system (Figure 9); this difference is due to the additional boundary conditions applied at $R=a$ for the free plate problem whereas the neglect of viscosity requires the elimination of two boundary conditions in the irrotational flow problem.

Of course, in real experiments of phase separated surfactant films, the domains are polydisperse in size, often interact, and may not be circular. To incorporate such effects, a statistical average over domain sizes and correlations in the scattered potential must be included. However, we expect the qualitative behavior of the simple models described here to be representative of the physical features of these systems. Furthermore, phase separating monolayers are rarely in equilibrium, the typical sizes and number density change after the ambient conditions are changed, and the time evolution of the film obeys complicated coarsening and topological relaxation mechanisms. It is not unreasonable that experimentally, long times need to elapse before reproducible damping results are attained. In fact, when scattering is important, capillary wave damping might be used as a diagnostic for probing the statistical averages and effective influences of phase separating morphologies.

The calculations presented for scattering with irrotational flows can be extended to include systems of membranes completely immersed in a fluid. Common examples are cell membranes, emulsions, or bilayers. As long as the geometry is symmetric top and bottom, both side contribute equally to the dynamical pressure; thus, the normal stress boundary condition becomes $\sigma(\vec{r}) \nabla_{\perp}^{2} \eta=2 \rho \partial_{t} \phi$. All the remaining steps follow with the substitution $\rho \rightarrow 2 \rho$. 
Results of scattering for such an immersed membrane are simply those for scattering at an air-liquid interface with $q_{0}, q_{1}$ rescaled by the modified $(\rho \rightarrow 2 \rho)$ dispersion relation $(2.20)$.

We have only considered a circular domain which affects the normal stress balance at the interface. However, the mathematical methods utilized in this paper can be extended to study tangential stress variations due to viscoelastic properties intrinsic to the film.16 Furthermore, a similar approach may be applicable for studying the effects of nonuniform surface stresses on the propagation of surface acoustic waves.

\section{ACKNOWLEDGMENTS}

T. Chou acknowledges Dionisios Margetis, Vassilios Houdzoumis, and David R. Nelson for their helpful discussions. H. A. Stone gratefully acknowledges a grant from NSF-PYI Award CT5-8957043.

\section{APPENDIX A: VISCOUS EFFECTS}

In this appendix we reconsider capillary wave scattering from a circular surface tension discontinuity in the presence of substrate fluid viscosity. We study solutions of the continuity equation $\nabla \cdot \vec{v}=0$ and the time-dependent Stokes equation, $\partial_{t} \vec{v}=-\nabla(p / \rho)+\nu \nabla^{2} \vec{v}$ for small amplitude capillary waves in an infinite depth fluid. Here, all variables $r, z$, and $t$ are dimensional. As before, the system is driven at a fixed frequency $\omega$ and all quantities are assumed to have an $e^{-i \omega t}$ time dependence. The limit of small viscous effects corresponds to the analysis presented in Section II.

When considering viscosity of the underlying liquid, tangential stresses at the interface must also be balanced. Accounting for the line tension $\gamma$ at the circular domain boundary and changes in surface tension $\sigma$, and neglecting any surface viscosity or elasticity of the surface film, the tangential stress balance has the form

$$
-\frac{\gamma}{a} \delta(r-a)+\vec{\nabla}_{\perp} \sigma(r)+\left.\rho \nu\left(\partial_{z} \vec{v}_{\perp}+\vec{\nabla}_{\perp} v_{z}\right)\right|_{z=0}=0 .
$$

For a step change of surface tension treated here (equation 2.9p), the discontinuity of $\nabla_{\perp} \sigma(\vec{r})$ at $r=a$ is balanced by the line tension term, $\frac{\gamma}{a} \delta(r-a)$ and the surface tangential stress condition obeyed everywhere on the interface is

$$
\left.\left(\partial_{z} \vec{v}_{\perp}+\vec{\nabla}_{\perp} v_{z}\right)\right|_{z=0}=0 .
$$

The normal stress balance including viscous effects is

$$
\sigma(\vec{r}) \nabla_{\perp}^{2} \eta=-p+\left.2 \rho \nu \partial_{z} v_{z}\right|_{z=0}
$$

Taking a time derivative of (A3) and using (2.3), we have

$$
\sigma(\vec{r}) \nabla_{\perp}^{2} v_{z}+2 i \rho \nu \omega \partial_{z} v_{z}=\left.i \omega p\right|_{z=0}
$$

To solve the unsteady Stokes equations, it is convenient to decompose the velocity fields as 16

$$
\vec{v}=\vec{\nabla} \phi(r)+\left(\begin{array}{c}
\vec{u}_{\perp} \\
u_{z}
\end{array}\right)
$$

where $\nabla^{2} \phi=0$, and $\vec{u}_{\perp}$ and $u_{z}$ satisfy the diffusion equation (recall all fields are proportional to $e^{-i \omega t}$ )

$$
-i \omega \vec{u}_{\perp}=\nu \nabla^{2} \vec{u}_{\perp} \quad-i \omega u_{z}=\nu \nabla^{2} u_{z}
$$

The pressure is $p=i \omega \rho \phi$.

We treat the incident $(i)$ and scattered $(s)$ waves separately by defining the incident fields, $\phi^{(i)}(x, z)$ and $\left(\vec{u}_{\perp}^{(i)}, u_{z}^{(i)}\right)$, and scattered fields, $\psi(r, \theta, z)$ and $\left(\vec{u}_{\perp}^{(s)}, u_{z}^{(s)}\right)$, such that

$$
\begin{aligned}
& \phi(r, \theta, z)=\phi^{(i)}(x, z)+\psi(r, \theta, z) \\
& \vec{u}_{\perp}=\vec{u}_{\perp}^{(i)}(x, z)+\vec{u}_{\perp}^{(s)}(r, \theta, z) \\
& u_{z}=u_{z}^{(i)}(x, z)+u_{z}^{(s)}(r, \theta, z) .
\end{aligned}
$$


The incident fields $\phi(x, z)$ and $\left(\vec{u}_{\perp}^{(i)}, u_{z}^{(i)}\right)$ determine the fluid velocity of a damped wave on a uniform interface of surface tension $\sigma_{0}$. These fields must satisfy the normal stress boundary condition (A4) with $\sigma=\sigma_{0}$ and the two-dimensional divergence of the tangential stress boundary condition (A3), respectively,

$$
\begin{aligned}
& \sigma_{0} \nabla_{\perp}^{2} u_{z}^{(i)}+\rho \omega^{2} \phi^{(i)}+2 i \omega \nu \partial_{z} u_{z}^{(i)}=0 \\
& 2 \partial_{z} \nabla_{\perp}^{2} \phi^{(i)}-\partial_{z}^{2} u_{z}^{(i)}+\nabla_{\perp}^{2} u_{z}^{(i)}=0 .
\end{aligned}
$$

Consider an incident wave with a velocity potential of unit amplitude on the $y$-axis, $x=z=0$. Substitution of $\phi^{(i)}(x, z)=e^{i Q_{0} x+Q_{0} z}$ and $u_{z}^{(i)}(x, z)=A e^{i Q_{0} x+l_{0} z}$, where $l_{0}^{2}=Q_{0}^{2}-i \omega / \nu$, into equation (A8) yields two equations for the unknowns $A$ and $Q_{0}(\omega)$ which are satisfied when

$$
A=-\frac{2 \nu Q_{0}^{3}}{2 \nu Q_{0}^{2}-i \omega}
$$

and

$$
\sigma_{0} Q_{0}^{3}-\rho \omega^{2}-4 \rho \nu^{2} Q_{0}^{2} l_{0}\left(Q_{0}+l_{0}\right)=0 .
$$

The condition (A10) determines the dispersion relation for capillary waves on a homogeneous surface with tension $\sigma_{0}$. In the $\nu \rightarrow 0$ limit, the first correction to the pole at $\sigma_{0} k_{0}^{3}=\omega^{2} a^{3}$ gives 19

$$
Q_{0} \approx k_{0}+i \epsilon \equiv k_{0}+i \frac{4 \omega \nu}{3 \sigma_{0}}
$$

which shows that incident waves are spatially damped by the factor $e^{\left(-4 \omega \nu / 3 \sigma_{0}\right) x}$.

We now consider scattering from a domain of radius $r=a$ and surface tension $\sigma_{1}$. The incident wave is the same as in the above nonscattering case, i.e., a wave that has unit velocity potential amplitude in the absence of the scatterer. The normal stress condition obeyed by the scattered fields is

$$
\begin{array}{lc}
\frac{\sigma_{0}}{\rho} \nabla_{\perp}^{2} u_{z}^{(s)}+\omega^{2} \psi+2 i \omega \nu \partial_{z} u_{z}^{(s)}=0 & (r>1) \\
\frac{\sigma_{1}}{\rho} \nabla_{\perp}^{2} u_{z}^{(s)}+\omega^{2} \psi+2 i \omega \nu \partial_{z} u_{z}^{(s)} & \\
\quad=-\frac{\sigma_{1}}{\rho} \nabla_{\perp}^{2} u_{z}^{(i)}-\omega^{2} \phi^{(i)}-2 i \omega \nu \partial_{z} u_{z}^{(i)} & (0 \leq r<1) .
\end{array}
$$

Since the tangential stress condition holds for all $r$, we can use (A2) to eliminate the $u_{z}^{(s)}$ terms from $($ A12) hence solving for $\psi$.

Expansions for the fields $\psi$ and $\left(\vec{u}_{\perp}, u_{z}\right)$ can be found which obey the Laplace and diffusion equations respectively. For each angular harmonic $\cos m \theta, \psi(r, \theta, z)$ can be written as a Hankel transform of $\psi_{m}(k) e^{k z}$ as in equation (2.11); similarly $u_{z}^{(s)}$ can be expanded in terms of $A_{m}(k) J_{m}(k r) e^{l z}$. Furthermore, with $\vec{u}_{\perp} \equiv\left(u_{r}, u_{\theta}\right)$, it is convenient to work with $u_{r}^{(s)} \pm u_{\theta}^{(s)}$, which are expanded in terms of $U_{m}^{ \pm}(k) J_{m \pm 1}(k r) e^{l z}$;

$$
\begin{gathered}
\psi(r, \theta, z)=\sum_{m=0}^{\infty} \cos m \theta \int_{0}^{\infty} d k \psi_{m}(k) J_{m}(k r) e^{k z} \\
u_{r}^{(s)}(r, \theta, z)=\sum_{m=0}^{\infty} \cos m \theta \int_{0}^{\infty} d k\left[U_{m}^{+}(k) J_{m+1}(k r)+U_{m}^{-}(k) J_{m-1}(k r)\right] e^{l z} \\
u_{\theta}^{(s)}(r, \theta, z)=\sum_{m=0}^{\infty} \sin m \theta \int_{0}^{\infty} d k\left[U_{m}^{+}(k) J_{m+1}(k r)-U_{m}^{-}(k) J_{m-1}(k r)\right] e^{l z}
\end{gathered}
$$

and

$$
u_{z}^{(s)}(r, \theta, z)=\sum_{m=0}^{\infty} \cos m \theta \int_{0}^{\infty} d k A_{m}(k) J_{m}(k r) e^{l z}
$$


where $l \equiv \sqrt{k^{2}-i \omega / \nu}$ and we must now determine the four functions $U_{m}^{ \pm}(k), A_{m}(k)$, and $\psi_{m}(k)$. Note the above integrands have a branch cut starting at $k=\sqrt{i \omega / \nu}$.

The remaining steps follow those described earlier though the algebraic details are more cumbersome. Substitution of (A13) and (A16) into equation (A2), multiplying the integrand by $\cos n \theta$ and integrating $\theta$ from 0 to $2 \pi$, we relate the two fields

$$
A_{n}(k)=-\frac{2 \nu k^{3}}{2 \nu k^{2}-i \omega} \psi_{n}(k)
$$

Applying the normal stress balance on the interface in the interior of the circular scatterer yields the dual integral equations of the same form as (2.16) where now we find the dimensionless functions

$$
F_{n}(q ; \tilde{Z})=\frac{i^{-n}}{a}\left(q_{0}^{3}-q^{3}+4 \tilde{Z}^{2} q^{2} m(q+m)\right) \psi_{n}(q / a)
$$

and

$$
G(q ; \tilde{Z})=\frac{q^{3}-q_{1}^{3}-4 \tilde{Z}^{2} \Lambda q^{2} m(q+m)}{q^{3}-q_{0}^{3}-4 \tilde{Z}^{2} \Lambda q^{2} m(q+m)},
$$

corresponding to (2.17) and (2.18) respectively, where the strength of the viscous effects are measured by the Ohnesorge number

$$
\tilde{Z}^{2}=\frac{\rho \nu^{2}}{\sigma_{0} a}
$$

We have used the definition (2.20) in the $h \rightarrow \infty$ limit for $q_{j}$ and defined the dimensionless quantity $m=l a=$ $\sqrt{q^{2}-q_{0}^{3 / 2} / \tilde{Z}}$. Also, the equivalent of the right side of the second equation in (2.16) is

$$
\Delta_{n}\left(Q_{0} ; \nu\right) J_{n}\left(Q_{0} r\right) \equiv\left(2-\delta_{n, 0}\right)(1-\Lambda)\left[\frac{Q_{0}^{3} a^{3}}{1+2 i Q_{0}^{2} a^{2} \tilde{Z} / q_{0}^{3 / 2}}\right] .
$$

Solving the the dual integral equations yields $F_{n}(q ; \tilde{Z})$ from which the $\psi_{n}(q / a)$ are determined. When $\tilde{Z}$ small, the capillary wave pole in $G(q ; \tilde{Z})$ is $q \approx Q_{0}$ as given in (A11). This positive imaginary shift when $\nu$ approaches zero fixes the integration path below the pole at $q \approx k_{0}$ in $(2.26)$. In the $\nu \rightarrow 0(\tilde{Z} \rightarrow 0)$ limit, this problem reduces to that of scattering of surface waves on an irrotational fluid of infinite depth.

The scattered velocity fields can be extracted by using the decompositions of the scattered fields, (A13 A16) to balance the $\hat{r}$ and $\hat{\theta}$ components of the tangential stress equation (A2). The remaining expansion coefficients $U_{n}^{ \pm}(k)$ are solved in terms of the $\psi_{n}(k)$ :

$$
U_{n}^{ \pm}(k)= \pm \frac{\nu k^{2} l}{2 \nu k^{2}-i \omega} \psi_{n}(k) .
$$

Adding the contributions from $\psi(r, \theta, z)$, the scattered velocities can be formally written as

$$
\begin{aligned}
& u_{r}^{(s)}(r, \theta, z)=\sum_{m=0}^{\infty} \cos m \theta \int_{0}^{\infty} d k\left[e^{k z}-\frac{2 \nu k l}{2 \nu k^{2}-i \omega} e^{l z}\right] \frac{d J_{m}(k r)}{d r} \psi_{m}(k) \\
& u_{\theta}^{(s)}(r, \theta, z)=\sum_{m=0}^{\infty} \sin m \theta \int_{0}^{\infty} d k\left[-e^{k z}+\frac{2 \nu k l}{2 \nu k^{2}-i \omega} e^{l z}\right] \frac{m}{r} J_{m}(k r) \psi_{m}(k) \\
& u_{z}^{(s)}(r, \theta, z)=\sum_{m=0}^{\infty} \cos m \theta \int_{0}^{\infty} k d k\left[e^{k z}-\frac{2 \nu k^{2}}{2 \nu k^{2}-i \omega} e^{l z}\right] J_{m}(k r) \psi_{m}(k) .
\end{aligned}
$$

To obtain the total velocity field of the scattering process, the incident velocities, $\vec{v}^{(i)}$, (corresponding to $\phi^{(i)}$ and $\left(u_{\perp}^{(i)}, u_{z}^{(i)}\right)$ in (A5)) must be added 


$$
\begin{gathered}
v_{x}^{(i)}=i Q_{0} e^{i Q_{0} x+Q_{0} z}-i \frac{Q_{0}}{l_{0}} \frac{2 \nu Q_{0}^{3}}{2 \nu Q_{0}^{2}-i \omega} e^{i Q_{0} x+l_{0} z} \\
v_{z}^{(i)}=Q_{0} e^{i Q_{0} x+Q_{0} z}-\frac{2 \nu Q_{0}^{3}}{2 \nu Q_{0}^{2}-i \omega} e^{i Q_{0} x+l_{0} z} .
\end{gathered}
$$

where $Q_{0}$ is given by the roots of $(\mathrm{A} 10) .19$

\section{APPENDIX B: BOUNDARY CONDITIONS FOR BENDING WAVES}

This Appendix tabulates intermediate results for the scattering produced by a circular inclusion with bending rigidity $D_{1}$ imbedded in a membrane with bending rigidity $D_{0}$. The four boundary conditions formulated in Section III B lead to four linear equations for the coefficients, $X_{i}=\left(A_{n}, B_{n}, C_{n}, E_{n}\right)$ which may be compactly written

$$
M_{i j} X_{j}=B_{i}
$$

where

$$
\mathbf{B}=i^{n}\left(2-\delta_{n, 0}\right)\left[\begin{array}{c}
J_{n}\left(q_{0}\right) \\
J_{n}^{\prime}\left(q_{0}\right) \\
\left(q_{0}^{2}+\left(\mu_{0}-2\right) n^{2}\right) J_{n}\left(q_{0}\right)-q_{0}\left(\mu_{0}-1\right) J_{n}^{\prime}\left(q_{0}\right) \\
n^{2}\left(\mu_{0}-1\right) J_{n}\left(q_{0}\right)+q_{0}\left(q_{0}^{2}-\mu_{0} n^{2}\right) J_{n}^{\prime}\left(q_{0}\right)
\end{array}\right]
$$

and $\mathbf{M}$ is the $4 \times 4$ matrix given by

$$
\begin{gathered}
M_{1 i}=\left(-H_{n}^{(1)}\left(q_{0}\right),-K_{n}\left(q_{0}\right), J_{n}\left(q_{1}\right), I_{n}\left(q_{1}\right)\right), \\
M_{2 i}=\left(-H_{n}^{(1) \prime}\left(q_{0}\right),-K_{n}^{\prime}\left(q_{0}\right), \frac{q_{1}}{q_{0}} J_{n}^{\prime}\left(q_{1}\right), \frac{q_{1}}{q_{0}} I_{n}^{\prime}\left(q_{1}\right)\right) \\
M_{31}=-\left[q_{0}^{2}+\left(\mu_{0}-2\right) n^{2}\right] H_{n}^{(1)}\left(q_{1}\right)+q_{0}\left(\mu_{0}-1\right) H_{n}^{(1) \prime}\left(q_{1}\right) \\
M_{32}=\left(q_{0}^{2}-\mu_{0} n^{2}\right) K_{n}\left(q_{0}\right)+q_{0}\left(\mu_{0}-1\right) K_{n}^{\prime}\left(q_{0}\right) \\
M_{33}=\frac{D_{1}}{D_{0}}\left[\left(q_{1}^{2}+\left(\mu_{1}-2\right) n^{2}\right) J_{n}\left(q_{1}\right)-q_{1}\left(\mu_{1}-1\right) J_{n}^{\prime}\left(q_{1}\right)\right] \\
M_{34}=-\frac{D_{1}}{D_{0}}\left[\left(q_{1}^{2}-\mu_{1} n^{2}\right) I_{n}\left(q_{1}\right)+q_{1}\left(\mu_{1}-1\right) I_{n}^{\prime}\left(q_{1}\right)\right] \\
M_{41}=-q_{0}\left(q_{0}^{2}-\mu_{0} n^{2}\right) H_{n}^{(1) \prime}\left(q_{0}\right)-n^{2}\left(\mu_{0}-1\right) H_{n}^{(1)}\left(q_{0}\right) \\
M_{42}=q_{0}\left[q_{0}^{2}+\left(\mu_{0}-2\right) n^{2}\right] K_{n}^{\prime}\left(q_{0}\right)-n^{2}\left(\mu_{0}-1\right) K_{n}\left(q_{0}\right) \\
M_{43}=\frac{D_{1}}{D_{0}}\left[q_{1}\left(q_{1}^{2}-\mu_{1} n^{2}\right) J_{n}^{\prime}\left(q_{1}\right)+\left(\mu_{1}-1\right) n^{2} J_{n}\left(q_{1}\right)\right] \\
M_{44}=-\frac{D_{1}}{D_{0}}\left[q_{1}\left(q_{1}^{2}+\left(\mu_{1}-2\right) n^{2}\right) I_{n}^{\prime}\left(q_{1}\right)-\left(\mu_{1}-1\right) n^{2} I_{n}\left(q_{1}\right)\right]
\end{gathered}
$$

$J_{n}^{\prime}(x)$, etc. denote the derivatives of Bessel functions.

The matrix $\mathbf{N}$ is identical to $\mathbf{M}$ except that the first column, $M_{i 1}$, is replaced by the column vector $\mathbf{B}$.

${ }^{1}$ C. M. Knobler and R. C. Desai, "Phase Transitions in Monolayers," Ann. Rev. Phys. Chem. 43, 207 (1992).

${ }^{2}$ H. M. McConnell, "Structures and Transitions in Lipid Monolayers at the Air-Water Interface," Ann. Rev. Phys. Chem. 42, 171 (1991).

${ }^{3}$ B. A. Noskov, "Dynamical Properties of Heterogeneous Surface Layers. Capillary Wave Scattering," Fluid Dynamics 26, 106 (1991).

${ }^{4}$ W. R. Dean, "On the reflexion of surface waves from a vertical barrier," Proc. Cambridge Phil. Soc. 41, 231 (1945).

${ }^{5}$ P. Devillard, F. Dunlop and B. Souillard, "Localization of gravity waves on a channel with a random bottom," J. Fluid Mech. 186, 521 (1988). 
${ }^{6}$ M. Belzons, E. Guazzelli and O. Parodi, "Gravity waves on a rough bottom: experimental evidence of one-dimensional localization," J. Fluid Mech. 186, 539 (1988).

${ }^{7}$ C.C. Mei, Applied Dynamics of Ocean Surface Waves (Wiley, New York, 1983).

${ }^{8}$ F. John, "On the motion of floating bodies," Comm. Pure and Appl. Math. 3, 45 (1950).

${ }^{9}$ D. Mahdmina, and L. M. Hocking, "Scattering of a Capillary-Gravity Wave by a Vertical Cylinder," Phys. Fluids A 2, 202 (1990).

${ }^{10}$ K. Miyano and K. Tamada, "Capillary wave propagation on water nonuniformly covered with a solid film," Langmuir 9, 508 (1993).

${ }^{11}$ Q. Y. Wang, A. Feder, and E. Mazur, "Capillary Wave Damping in Heterogeneous Monolayers," J. Phys. Chem. 98, 12720, (1994).

${ }^{12}$ E. H. Lucassen-Reynders and J. Lucassen, "Properties of capillary waves," Advances in Coll. Int. Sci. 2, 331 (1969).

${ }^{13}$ Y. L. Chen, M. Sano, M. Kawaguchi, H. Yu, and G. Zografi, "Static and Dynamic Properties of Pentadecanoic Acid Monolayers at the Air-Water Interface," Langmuir 2, 349 (1986).

${ }^{14}$ C. Stenvot and D. Langevin, "Study of Viscoelasticity of Soluble Monolayers Using Analysis of Propagation of Excited Capillary Waves," Langmuir 4, 1179 (1988).

${ }^{15}$ K. Y. Lee, T. Chou, and D. S. Chung, and E. Mazur, "Direct Measurement of the Spatial Damping of Capillary Waves at Liquid-Vapor Interfaces," J. Phys. Chem. 97, 12876 (1993).

16 T. Chou and D. R. Nelson, "Water Wave Scattering at Nonuniform Fluid Interfaces," J. Chem. Phys. 101, 9022 (1994).

17 A. E. Heins, "The Scope and Limitations of the Method of Weiner and Hopf," Comm. Pure and Appl. Math. 9, 447 (1956).

${ }^{18}$ S. Gou, A. F. Messiter and W. W. Schultz, "Capillary-gravity waves in a surface tension gradient. I: Discontinuous change," Phys. Fluids A 5, 966 (1993).

${ }^{19}$ V. G. Levich, Physicochemical Hydrodynamics (Prentice-Hall, Englewood Cliffs, N.J., 1962).

${ }^{20}$ I. S. Gradshteyn and I. M. Ryzhik, Table of Integrals, Series, and Products, (Academic Press, Inc., San Diego, 1980).

${ }^{21}$ G. Arfken, Mathematical Methods for Physicists (Academic Press, Inc., San Diego, 1985).

${ }^{22}$ C. J. Tranter, Integral Tranforms in Mathematical Physics (John Wiley \& Sons, 1966).

${ }^{23}$ G. N. Watson, The Theory of Bessel Functions, $2^{\text {nd }}$ Ed. (Cambridge University Press, Cambridge, 1944).

${ }^{24}$ S. K. Lucas, "Evaluating infinite integrals involving products of Bessel functions of arbitrary order," to appear in J. Comput. Appl. Math. (1994).

${ }^{25}$ A. G. Petrov and I. Bivas, "Elastic and Flexoelectic Aspects of Out-of-Plane Fluctuations in Biological and Model Membranes," Prog. Surface Sci. 16, 389 (1984).

${ }^{26}$ L. D. Landau and E. M. Lifshitz, Theory of Elasticity, $3^{\text {rd }}$ ed. (Pergamon Press, New York,1989).

${ }^{27}$ W. Soedel, Vibrations of Shells and Plates (Marcel Dekker, Inc., New York, 1981).

${ }^{28}$ P. M. Morse and K. U. Ingard, Theoretical Acoustics (Princeton University Press, Princeton, NJ, 1986).

${ }^{29}$ Results of Reference (9) also show similar oscillatory behavior in $\Sigma$.

${ }^{30}$ H. C. Van De Hulst, Light Scattering by Small Particles (John Wiley and Sons, Inc., New York, 1957).

31 Thermal fluctuations of membranes can often renormalize Poisson's ratios and drive them negative. See L. Radzihovsky, "Statistical Mechanics and Geometry of Random Manifolds," Ph.D. Thesis, Harvard University, 1993.

${ }^{32}$ A. Ishimaru, Wave Propagation and Scattering in Random Media, v. 2 (Academic Press, New York, 1978).

${ }^{33}$ J. Meunier, D. Langevin, and N. Boccara, Eds., Physics of Amphiphilic Layers (Springer Verlag, Berlin, 1987). 
FIG. 1. (a) A membrane with a circular domain of surface tension $\sigma_{1}$ imbedded in an interface of tension $\sigma_{0}$. (b) A plate of thickness $d$ and flexural rigidity $D_{0}$ with a circular inclusion of rigidity $D_{1}$. Ripples in the surface represent the membrane fluctuations due to an incident plane wave and the scattered wave.

FIG. 2. The contour used to evaluate $L_{m l}^{(n)}$ and $\psi(r, \theta, z)$. An infinitesimally small positive viscosity and imposition of an outgoing solution requires that either the pole requires a small positive imaginary part, or that we take the contour through a semicircle below the pole.

FIG. 3. Scattering amplitudes $|f(\theta)|$ for an infinite depth system with $\Lambda=\sigma_{0} / \sigma_{1}=2$. The same plots for the uncoupled membrane, equation (2.50), at $T_{0} / T_{1}=2^{2 / 3}$ are nearly identical.

FIG. 4. The scattering amplitudes $|f(\theta ; h)|$ for various depths $h=\infty, 0.5,0.1$ at $q_{0}=1,2,3,4$ for $\Lambda=2$. A "+" identifies the origin.

FIG. 5. (a) Total scattering cross section $\Sigma\left(q_{0}, \Lambda=2, h\right)$ for the surface tension discontinuity problem. Four depths are considered: $h=\infty, 0.2,0.1$, and 0.01. (b) Total scattering cross section $\Sigma\left(q_{0}, \Lambda=10, h\right)$ for $h=\infty, 1$, and 0.1 . (c) A comparison of the scattering cross section calculated numerically and asymptotically in the small $q_{0}$ limit for $\Lambda=1.1$. In both (a) and (b) the dark dashed curve is $\Sigma$ in the free membrane case, Section IIE.

FIG. 6. Depth dependence of $\Sigma\left(q_{0}, \Lambda=2, h\right)$ for scattering from a circular surface tension variation at incoming wavevectors, $q_{0}=1.0,2.0,3.0,4.0,5.0,8.0,9.0$, and 10.0

FIG. 7. $\Lambda$ dependence of $\Sigma\left(q_{0}, \Lambda, h=\infty\right)$ on surface tension ratios. For $\Lambda^{-1}>1$, mechanical stability requires an externally imposed negative line tension. The limit $\Lambda^{-1} \rightarrow \infty$ corresponds to an unstretchable domain.

FIG. 8. The scattering amplitudes for an interface with bending rigidity; $h=\infty, 0.5,0.1$ and $\Lambda_{d}=2^{5 / 3}$. The dotted curves are scattering from a free elastic plate. In this case, the Poisson's ratios are $\mu_{0}=0.25, \mu_{1}=-0.25$.

FIG. 9. Total cross sections of bending wave scattering for fluid depths $h=\infty, 0.2,0.1,0.01$. The dotted line is for a free plate with $\mu_{0}=0.25, \mu_{1}=-0.25$. 


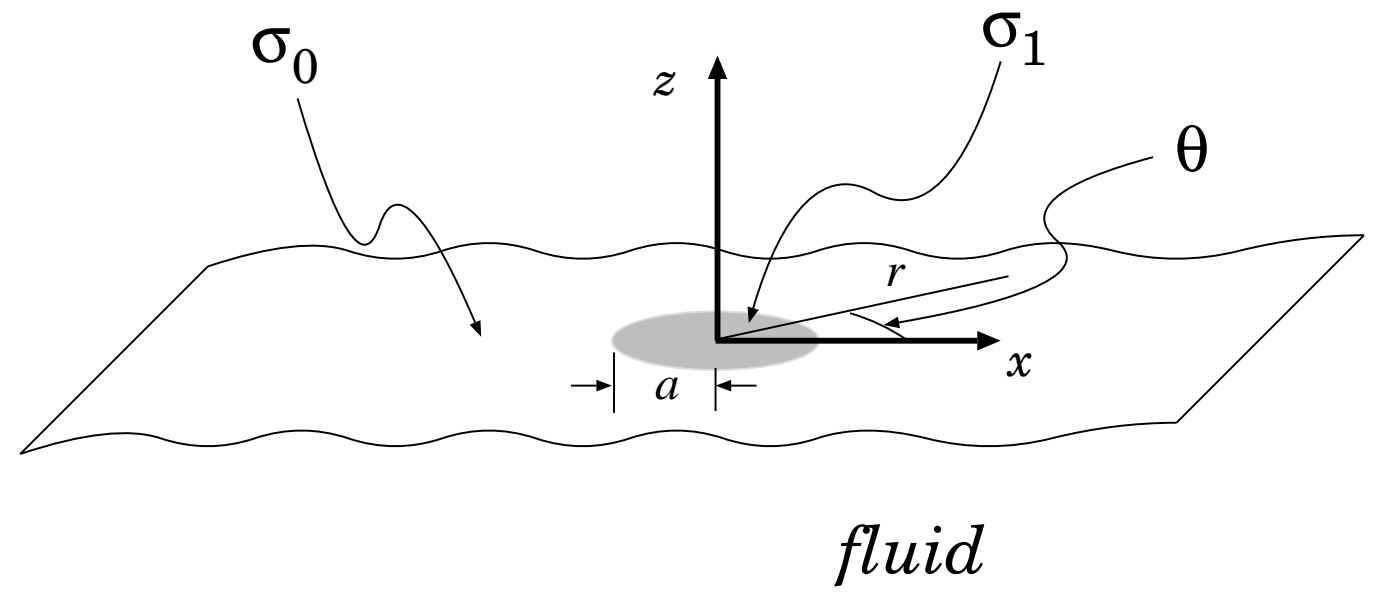

(a)

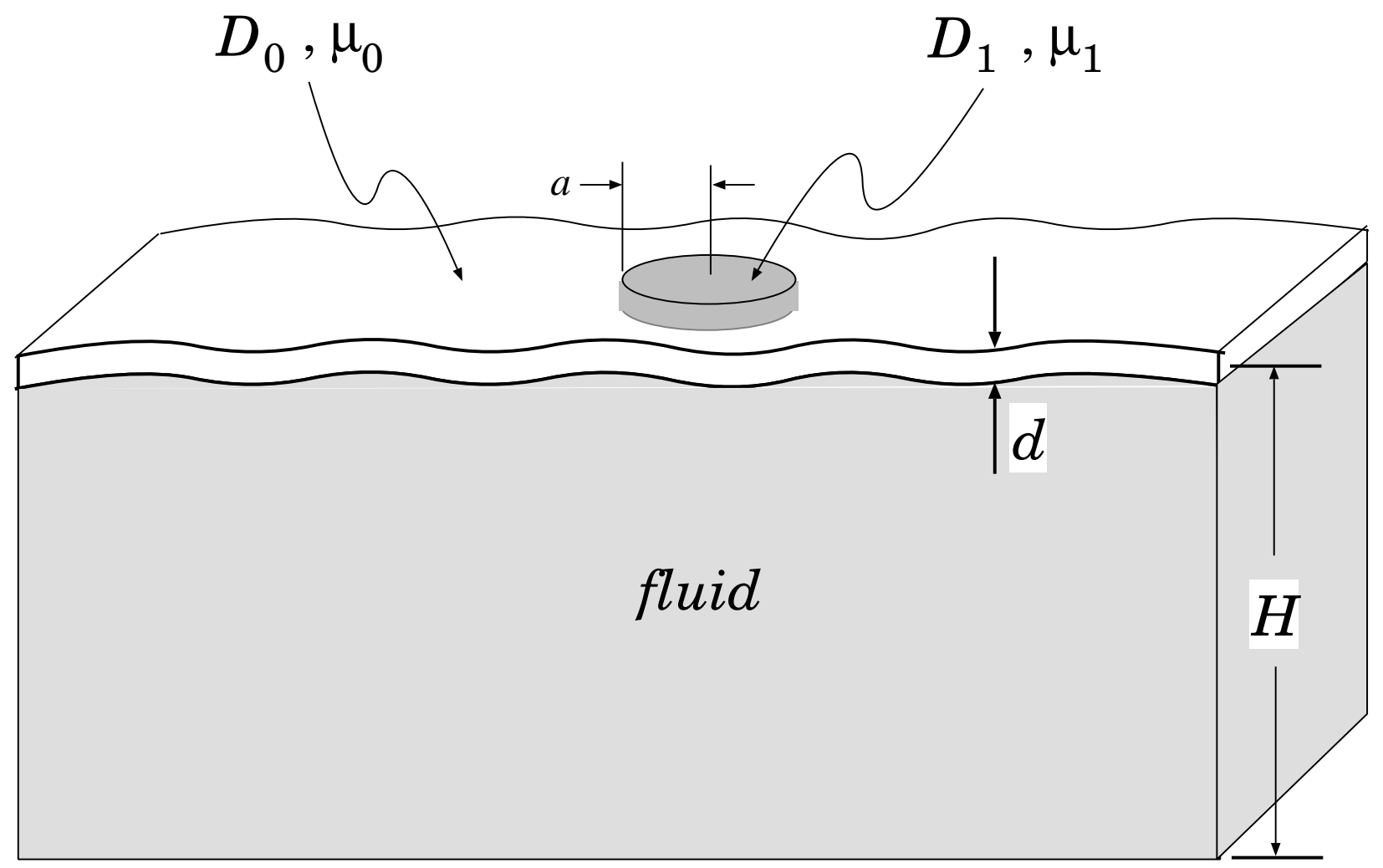

(b)

Figure 1

Chou, Lucas, and Stone 


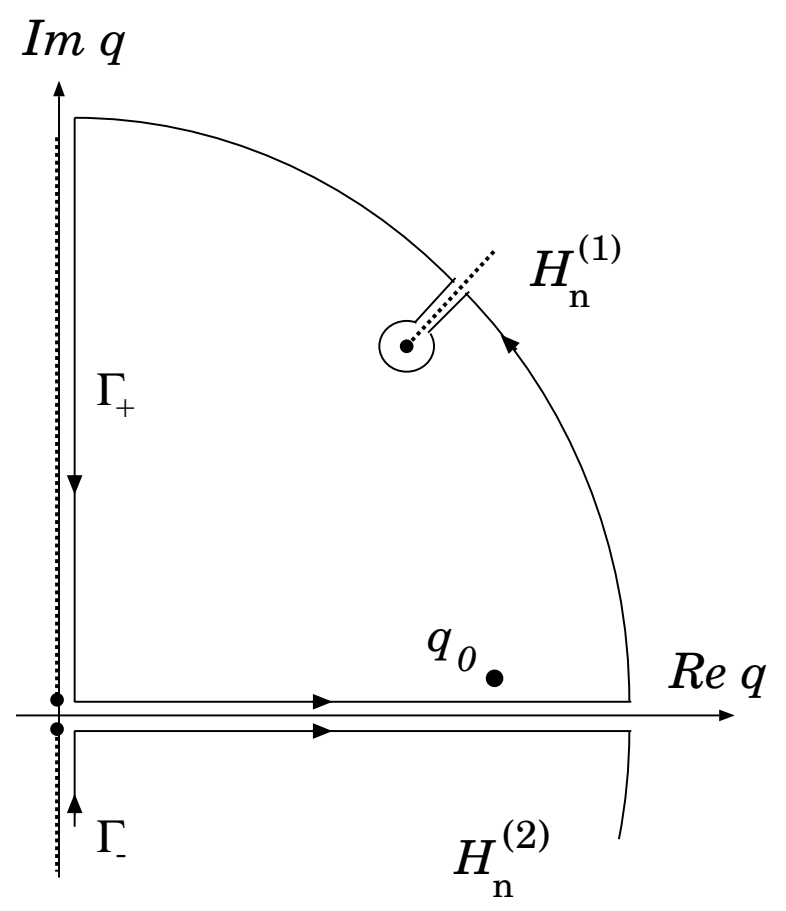

Figure 2

Chou, Lucas, and Stone 


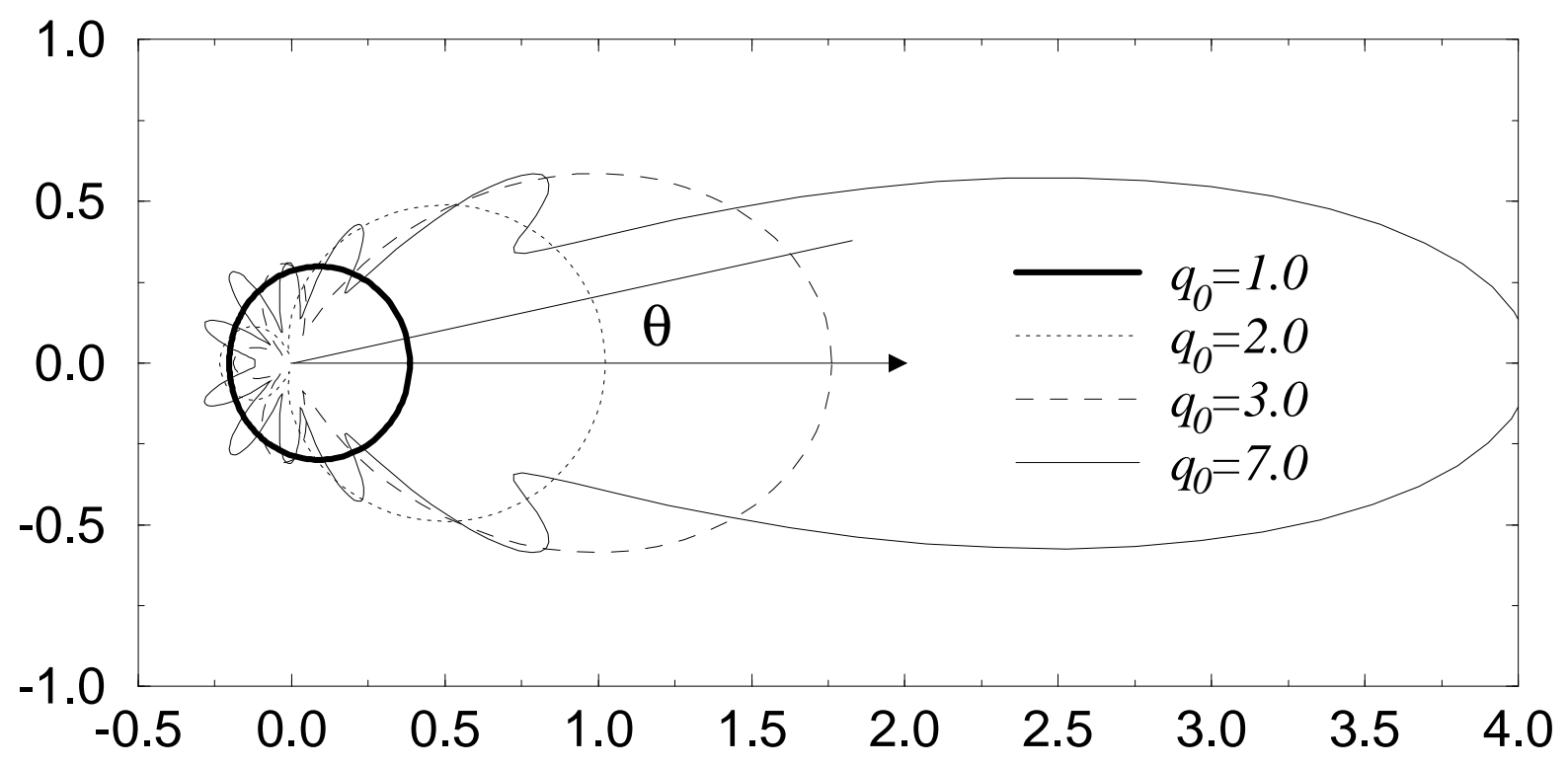

Figure 3

Chou, Lucas, and Stone 

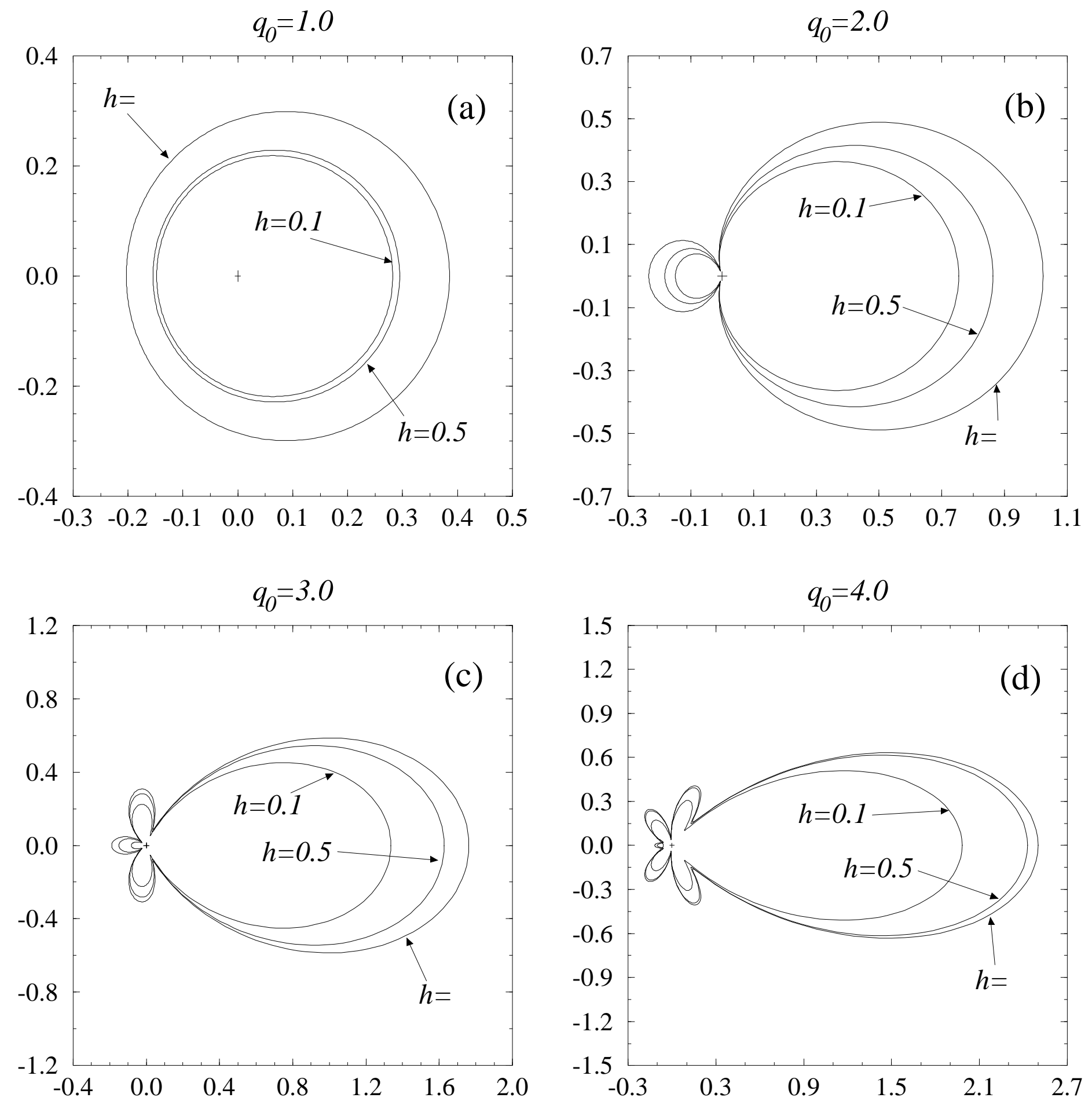

Figure 4 
(a)

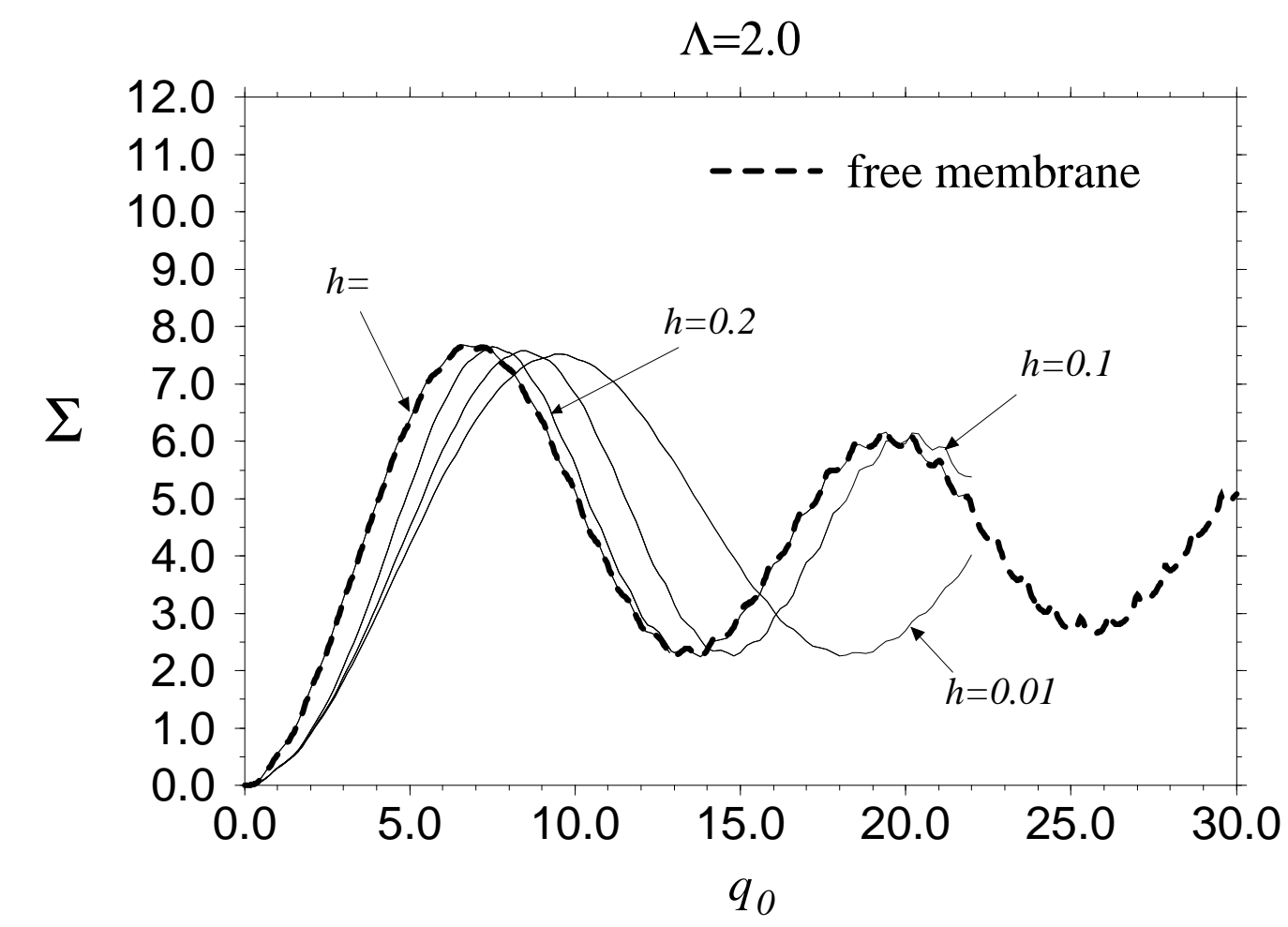

(b)

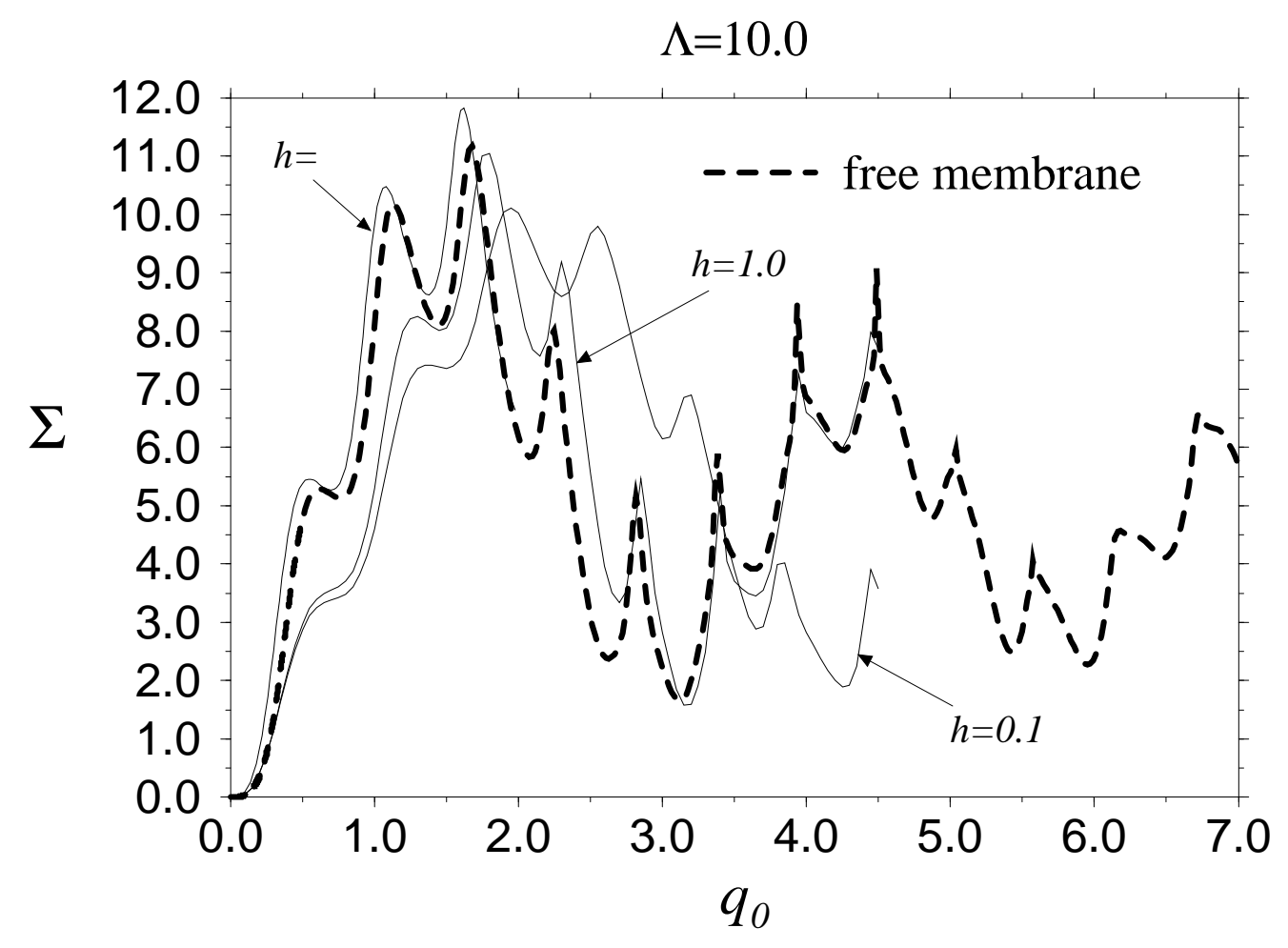

Figure 5 


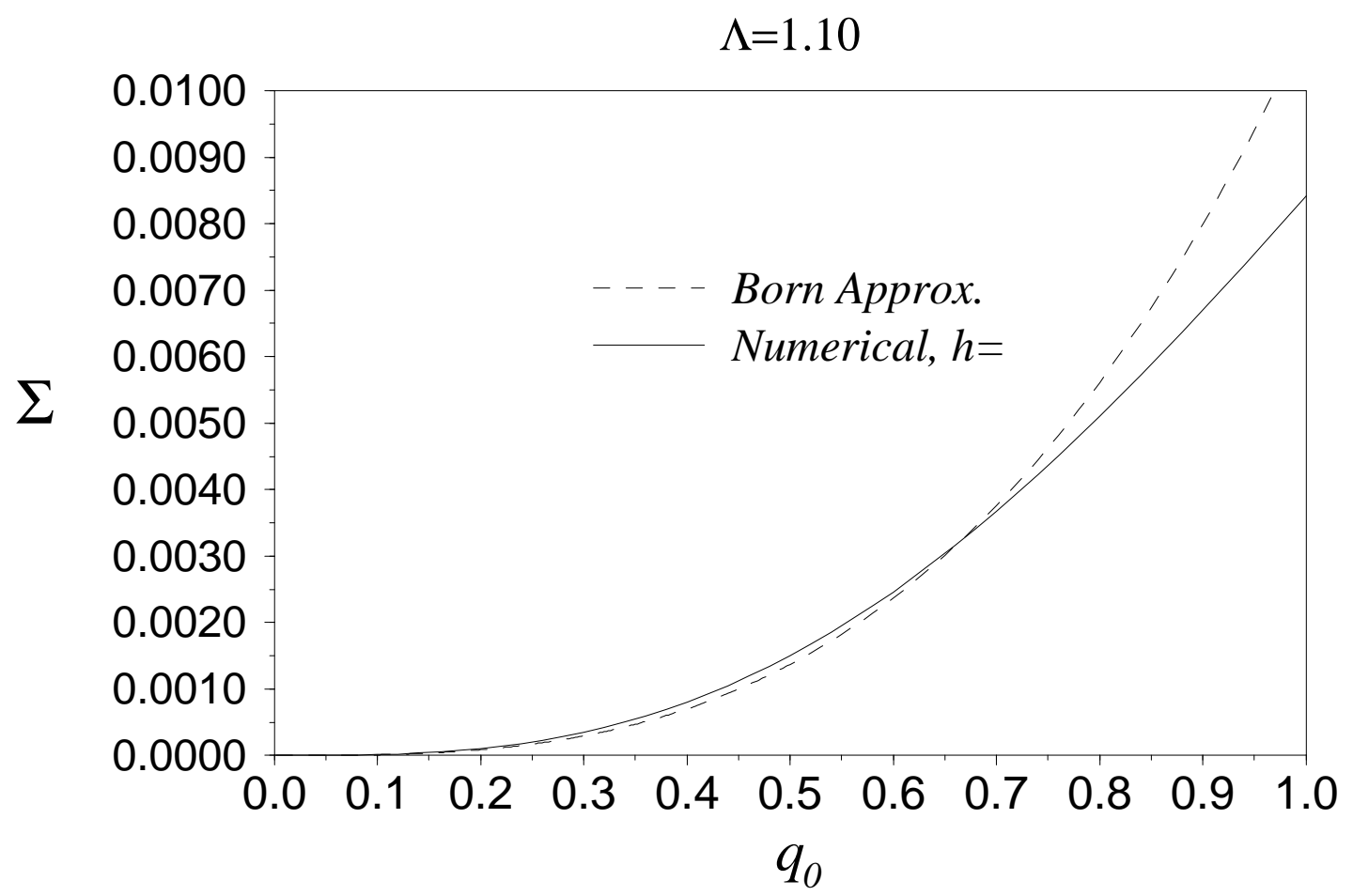

(c)

Figure 5(c) 


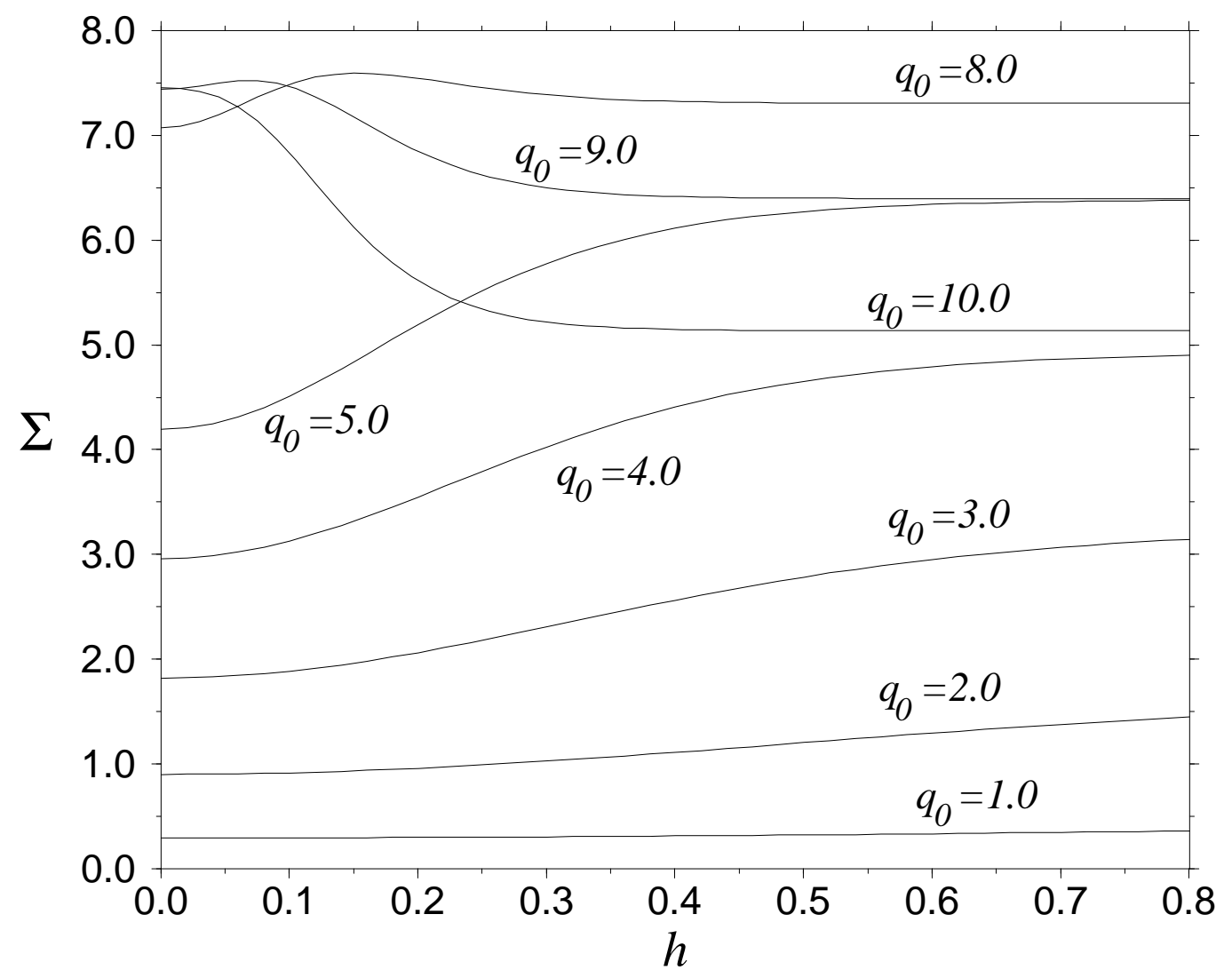

Chou, Lucas, and Stone 


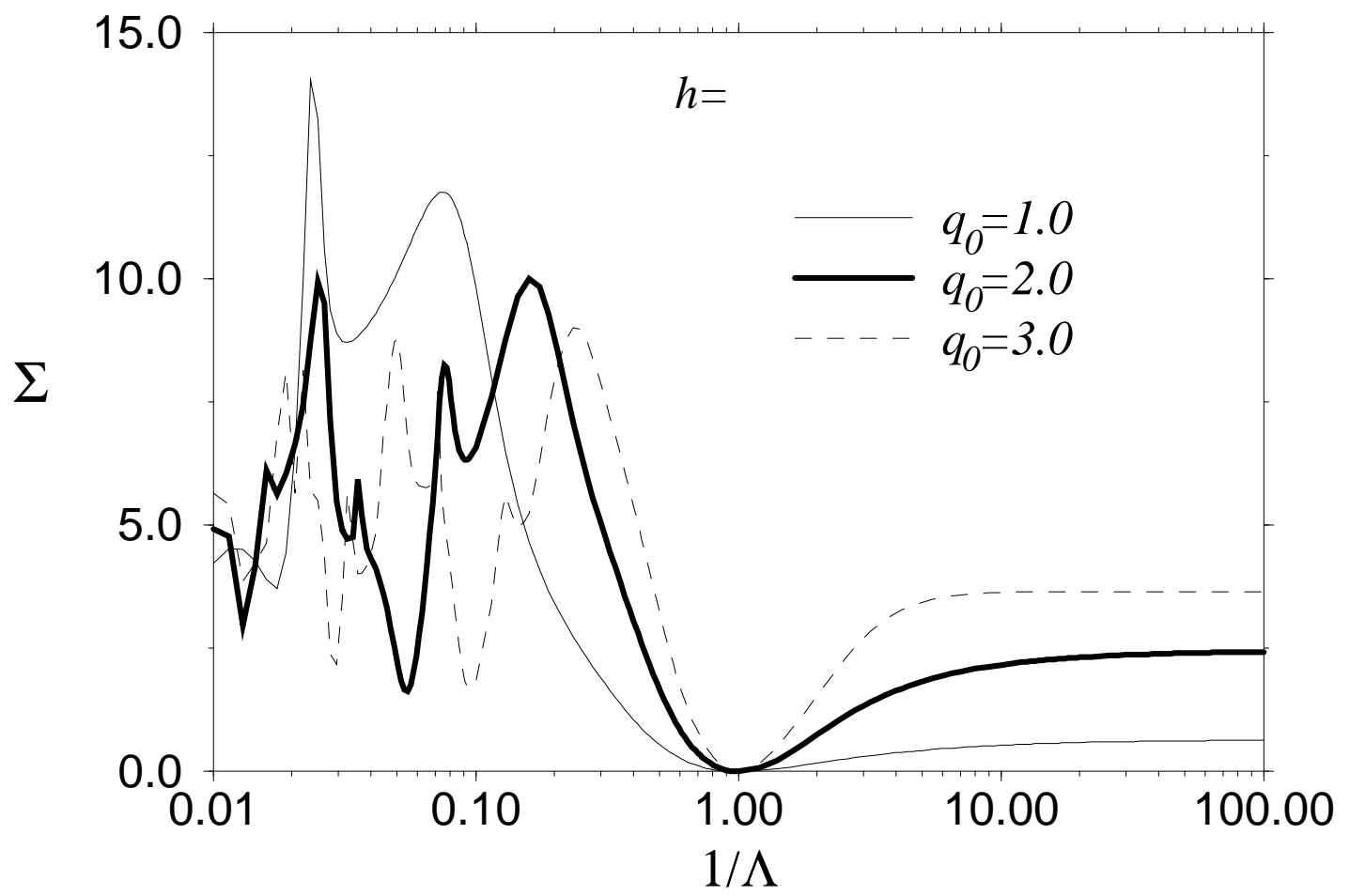

Figure 7

Chou, Lucas, and Stone 

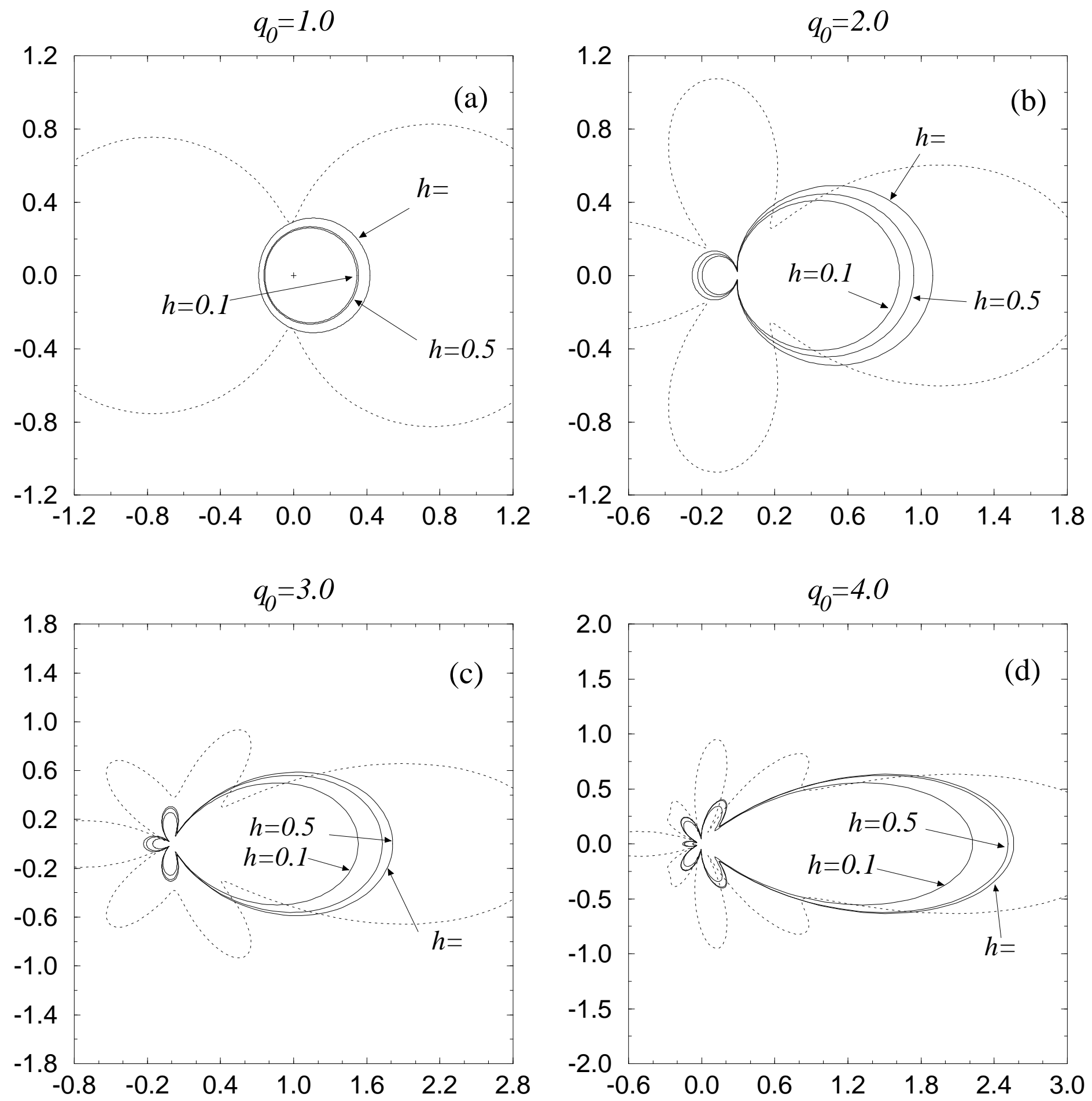

Figure 8 